Aus der Abteilung Allgemein-, Viszeral- und Kinderchirurgie (Prof. Dr. med. M. Ghadimi)

der Medizinischen Fakultät der Universität Göttingen

\title{
Management der nekrotisierenden Pankreatitis - Stellenwert der Kolektomie
}

\author{
INAUGURAL - DISSERTATION \\ zur Erlangung des Doktorgrades für Zahnheilkunde \\ der Medizinischen Fakultät der \\ Georg-August-Universität zu Göttingen
}

vorgelegt von

Marieke Helene Thomsen

aus

Kiel 
Dekan:

1. Berichterstatter:

2. Berichterstatter:

3. Berichterstatter:
Prof. Dr. rer. nat. H. K. Kroemer

Prof. Dr. med. O. Kollmar

PD Dr. med. A. Amanzada

Prof. Dr. med. L. Trojan

Tag der mündlichen Prüfung: 14. März 2016 


\section{Inhaltsverzeichnis}

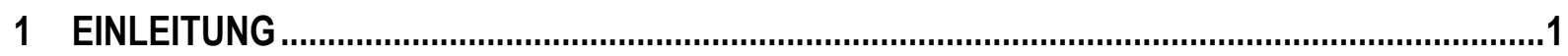

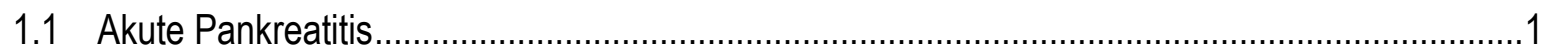

1.1.1 Definition und Stadieneinteilung ..................................................................................

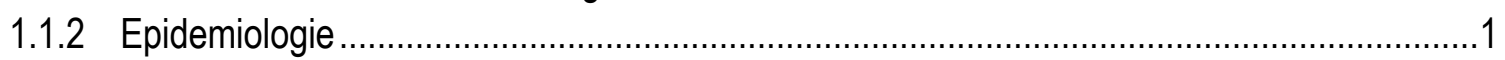

1.1.3 Ätiologie und Risikofaktoren ....................................................................................2

1.1.4 Pathogenese und Pathophysiologie .............................................................................2

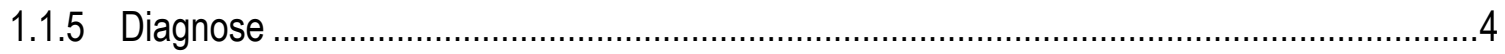

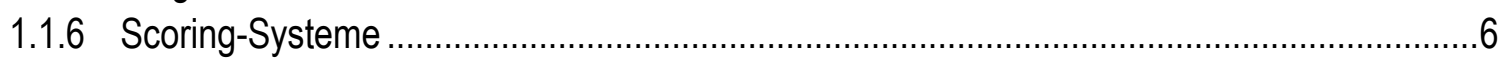

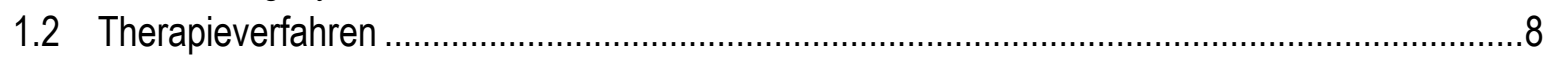

1.2.1 Konservative Therapie ...........................................................................................

1.2.2 Stellenwert der Chirurgie in der Behandlung der akuten nekrotisierenden Pankreatitis .....10

1.2.2.1 Chirurgische Therapie zur Behandlung der Ursachen der Pankreatitis ......................10

1.2.2.2 Chirurgische Therapie bei akuter nekrotisierender Pankreatitis ohne Kolektomie........11

1.2.2.3 Konzept der kombinierten Nekrosektomie und Kolektomie........................................12

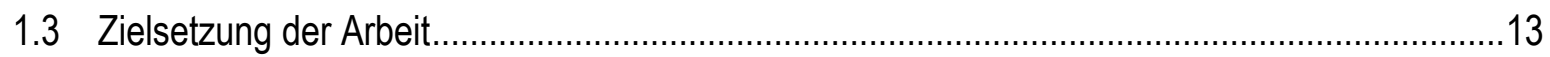

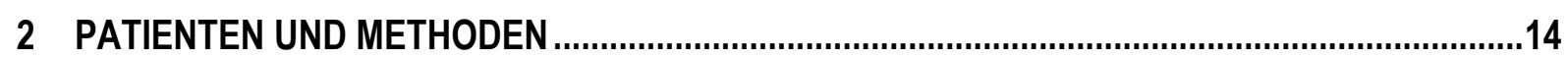

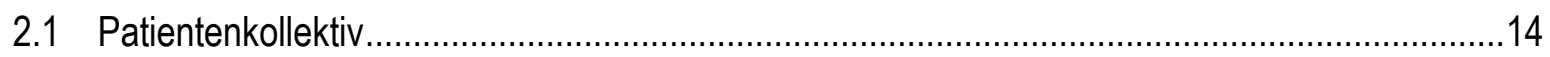

2.2 Datenerhebung / Methoden ................................................................................................15

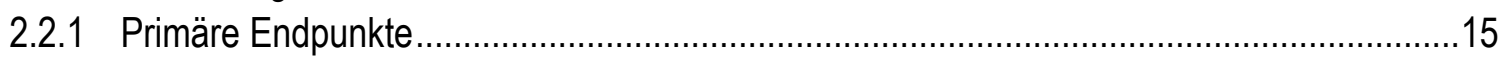

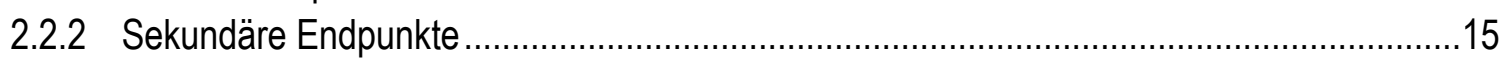

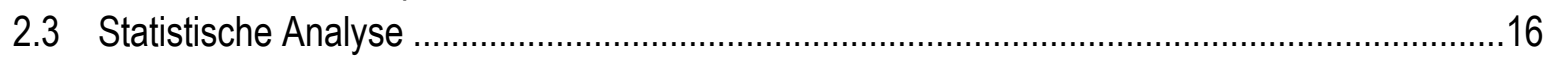

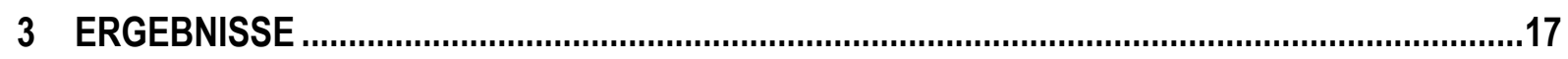

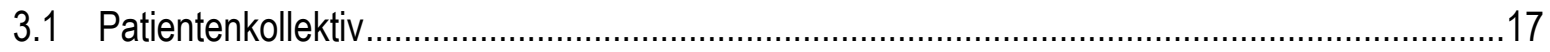

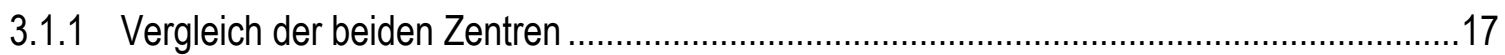

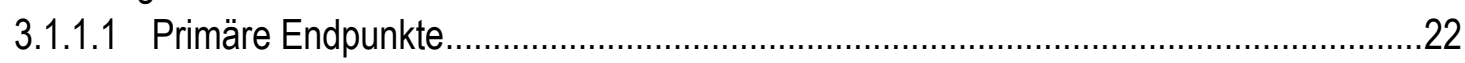

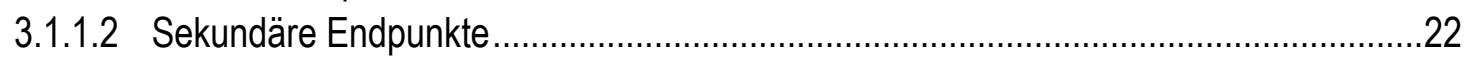

3.1.2 Subgruppenvergleich der unterschiedlichen Therapieoptionen .......................................23

3.1.2.1 Einfluss der verschiedenen chirurgischen Therapiekonzepte ..................................23

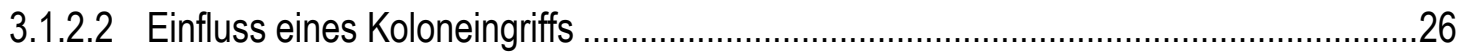

3.1.2.3 Radikales Vorgehen: Einfluss der Kolektomie .........................................................27

3.1.2.4 Auswirkung der Durchführung einer Kolektomie im Rahmen des Ersteingriffs ............28

3.1.2.5 Stellenwert einer frühzeitigen Kolektomie ............................................................29

3.1.2.6 Zentrumsübergreifende Analyse des Stellenwertes der Kolektomie ............................31

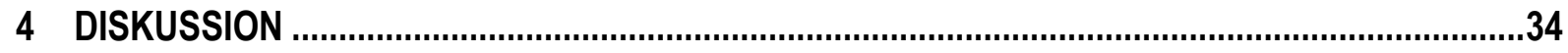

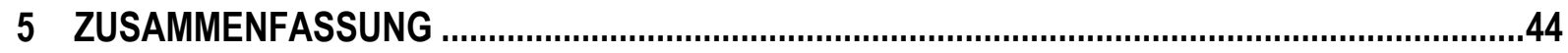

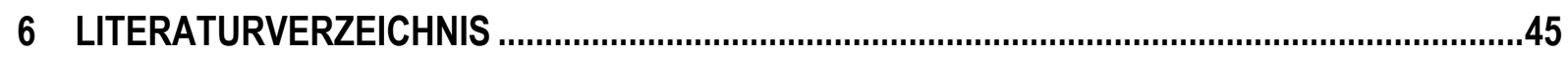

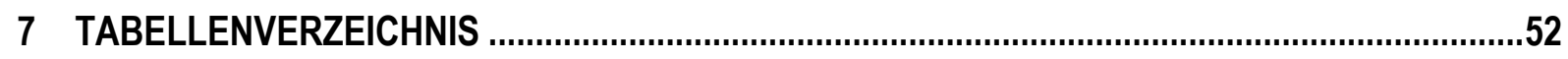

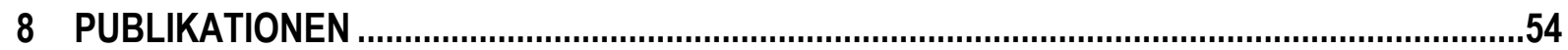




\section{Abkürzungsverzeichnis}

\begin{tabular}{|c|c|}
\hline ANV & akutes Nierenversagen \\
\hline AP & alkalische Phosphatase \\
\hline APACHE & acute physiology and chronic health evaluation \\
\hline ARDS & acute respiratory distress syndrome \\
\hline CRP & C-reaktives Protein \\
\hline CT & Computertomographie \\
\hline $\mathrm{DIC}$ & disseminierte intravasale Koagulopathie \\
\hline EKG & Elektrokardiogramm \\
\hline ERCP & endoskopisch retrograde Cholangiopankreatikographie \\
\hline GÖ & Universitätsklinikum Göttingen \\
\hline GOT & Glutamat-Oxalacetat-Transaminase \\
\hline GPT & Glutamat-Pyruvat-Transaminase \\
\hline $\mathrm{HOM}$ & Universitätsklinikum Homburg/Saar \\
\hline ICD-10-Codierung & $\begin{array}{l}\text { international statistical classification of diseases and related health problems; } \\
\text { Version } 2012\end{array}$ \\
\hline IKPM & amtlicher Operationenschlüssel \\
\hline $\mathrm{KM}-\mathrm{CT}$ & kontrastmittelverstärkte Computertomographie \\
\hline $\mathrm{LDH}$ & Lactatdehydrogenase \\
\hline MODS & multiple organ dysfunction syndrome \\
\hline MRT & Magnetresonanztomographie \\
\hline $\mathrm{PaO}_{2}$ & arterieller Sauerstoffpartialdruck \\
\hline SIRS & systemic inflammatory response syndrome \\
\hline$y-G T$ & Gamma-Glutamyltransferase \\
\hline
\end{tabular}




\section{$1 \quad$ Einleitung}

\subsection{Akute Pankreatitis}

\subsubsection{Definition und Stadieneinteilung}

Bei der akuten Pankreatitis handelt es sich um eine akute entzündliche Erkrankung, die durch einen autodigestiven Prozess des Organs charakterisiert ist. Dieser wird durch eine unkontrollierte intrazelluläre Aktivierung von den in den Azinuszellen gebildeten Verdauungsenzymen ausgelöst.

Die aktuelle Atlanta-Klassifikation von 2012 unterscheidet die akute Pankreatitis nach ihrem zeitlichen Verlauf in eine frühe (in den ersten 1-2 Wochen) und eine späte Phase. Außerdem unterteilt sie die Erkrankung nach dem Ausmaß der Parenchymzerstörung in eine milde, interstitiell-ödematöse (80 \%) und eine schwere, hämorrhagisch-nekrotisierende Form (20 \%) (Banks et al. 2013, Sarr 2013). Während die milde Form in der Regel ohne bleibende Schäden ausheilt, ist die schwere akute Pankreatitis meist mit langwierigen und komplizierten Verläufen assoziiert. Die hohe Sterblichkeit der schweren akuten Pankreatitis ist am ehesten auf infektiöse Komplikationen im Bereich nekrotischer Pankreasanteile sowie auf sekundäre Organschäden im Rahmen der mit der schweren nekrotisierenden Pankreatitis einhergehenden systemischen Erkrankung zurückzuführen (Gurusamy et al. 2005).

Die Gesamtletalität ist stark abhängig vom Schweregrad der Erkrankung und variiert zwischen 4-7 \% aller Fälle und 20-50 \% der Fälle, bei denen eine schwere Form diagnostiziert wurde (Bank et al. 2002). Im Vergleich zur Häufigkeit der Neuerkrankungen ist die Letalität in den letzten Jahrzehnten deutlich gesunken. Zurückzuführen ist dies auf verschiedene Faktoren, wie beispielsweise die Verwendung von Risikoprädiktion (Ranson-Kriterien, APACHE-II-Score siehe 1.1.6), durch deren Einführung der Schweregrad der Erkrankung besser abgeschätzt und umgehend eine entsprechende stadiengerechte/adaptierte Therapie eingeleitet werden kann, die computertomographische Diagnostik und verbesserte intensivmedizinische Therapie (Bank et al. 2002).

\subsubsection{Epidemiologie}

Die Inzidenz in Deutschland beträgt 19,7 auf 100.000 Einwohner pro Jahr. Männer sind 1,5 mal häufiger betroffen als Frauen (Lankisch et al. 2002). 
In einer Studie zeigte sich, dass das Geschlecht durchaus kein unabhängiger Risikofaktor für das Ergebnis bei akuter Pankreatitis ist. So konnte zwar im Hinblick auf den Schweregrad der Erkrankung kein signifikanter Zusammenhang ermittelt werden, wohl aber ein Unterschied in Ätiologie und Verlauf (Lankisch et al. 2001a). In den USA stellt die akute Pankreatitis den dritthäufigsten gastroenterologischen Grund für eine stationäre Behandlung dar und verursacht jährlich Kosten von mehr als 2 Mrd. US-\$ (Fagenholz et al. 2007, Shaheen et al. 2006).

Während der Erkrankungsgipfel für andere schwerwiegende pankreatische Erkrankungen wie die chronische Pankreatitis bei 45-54 Jahren oder das Pankreas-Karzinom bei 65-75 Jahren liegt, erkranken die meisten Menschen an der akuten Form der Pankreatitis bereits weitaus früher, nämlich zwischen dem 35. und 44. Lebensjahr (Lankisch et al. 2002).

\subsection{3 Ätiologie und Risikofaktoren}

Die Ursachen für die akute Pankreatitis sind heterogen. Gallensteinleiden (38\%) und Alkoholabusus (36 \%) sind in den Industrieländern die häufigsten Ursachen für eine akute Pankreatitis (Lankisch et al. 2001a, Spanier et al. 2008).

Weitaus seltener ist eine akute Pankreatitis als Komplikation bei endoskopisch retrograder Cholangiopankreatikographie (ERCP). Akute Pankreatitiden infolge von Virusinfektionen (Mumps), abdominellen Traumata, Stoffwechselerkrankungen wie Hyperlipidämie, medikamentös indiziert oder auch als Ausschlussdiagnose „idiopathisch“ stellen Sonderformen dar und sind sehr selten (Gupta und Wu 2010, Mitchell et al. 2003).

\subsubsection{Pathogenese und Pathophysiologie}

Mit ihrer sowohl endokrinen als auch exokrinen Funktion stellt die Bauchspeicheldrüse eine Schlüsselrolle im Stoffwechsel des menschlichen Körpers dar.

Die Pathogenese der Entzündung ist bislang nicht abschließend geklärt und bleibt weiterhin Gegenstand vieler Untersuchungen. Die zu der Entzündung führenden Abläufe auf molekularer Ebene werden erst teilweise verstanden und werden daher kontrovers diskutiert.

Allgemein anerkannt ist die Theorie, dass es durch die ätiologischen Faktoren wie Alkohol, Toxine oder intrapankreatische Drucksteigerung durch Gallensteine zu zellulärem Stress kommt, in dessen Folge der Transport der zymogenhaltigen Granula an die apikale Membran der Azinuszellen gestört wird. Diese Granula verschmelzen daher intrazellulär mit den Lysosomen, die zahlreiche hydrolytische Enzyme enthalten. Es kommt so zu einer vorzeitigen intrazellulären, intrapankreatischen Aktivierung 
von Trypsin. Trypsin ist eine unspezifische Endopeptidase und aktiviert in der Folge eine intrazelluläre Kaskade aus Phospholipasen und Elastase und initiiert sowohl den autodigestiven Prozess wie auch eine Migration von Neutrophilen in das Pankreas. Die autolytische Schädigung des Pankreasparenchyms wird zudem durch die infiltrierenden Granulozyten und die durch diese freigesetzten Zytokine, Sauerstoffradikale und lytischen Enzyme verstärkt (Mossner 2011, Wang et al. 2009). Die massive Freisetzung der pro-inflammatorischen Zytokine im Pankreas führt zu einer Störung der lokalen anti-inflammatorischen Regulationsmechanismen (Proteaseinhibitoren, Antiproteasen).

Das Ausmaß der lokalen Entzündungsreaktion sowie die Schwere der Dysregulation der lokalen antiinflammatorischen Mechanismen entscheiden, ob die Pankreatitis nach serös-exsudativer Verlaufsform ausheilt oder in eine nekrotisierende Verlaufsform übertritt. Experimentell konnte gezeigt werden, dass Mikrozirkulationsstörungen im Rahmen der lokalen Entzündungsreaktion in Form von kapillärem Perfusionsversagen zu einer lokalen Hypoxie oder Anoxie führen und zusammen mit der inflammatorischen Gewebeschädigung zur Ödembildung und Nekroseentwicklung beitragen (Menger et al. 2001). Darüber hinaus bewirkt die lokale Entzündungsreaktion ein Zusammenbrechen der Endothelbarriere mit konsekutivem Gewebsödem sowie eine systemische Ausbreitung der im Rahmen der Azinuszell-Nekrose freigesetzten pankreatischen Enzyme. Darüber hinaus werden die im Rahmen der lokalen Entzündungsreaktion freigesetzten Zytokine in die systemische Zirkulation freigesetzt. Diese systemische Zytokinämie führt in der Frühphase der akuten Pankreatitis zu einer Dysregulation der systemischen Immunantwort (Rau et al. 2000).

Zudem können im Rahmen der systemischen Ausbreitung der Entzündungsreaktion (SIRS) andere Organsysteme massiv geschädigt werden, was sich in Form von pulmonalen, kardiovaskulären und renalen Insuffizienzen als sekundäre Organschädigungen manifestiert (Johnson et al. 2001).

Klinisch zeigt sich ein mehrphasiger Krankheitsverlauf. Die akute Pankreatitis ist durch akut einsetzende, gürtelförmige Oberbauchschmerzen charakterisiert. Innerhalb der ersten 48 Stunden entwickelt sich resultierend aus der systemischen Störung der Endothelbarriere eine massive Flüssigkeitsverschiebung ins Interstitium mit intravasaler Hypovolämie. Bei der Mehrzahl dieser Patienten zeigen sich im CT bereits zu diesem Zeitpunkt intra- und extrapankreatische Nekrosen (Isenmann und Beger 1999).

Die bakterielle Kontamination der Nekroseareale charakterisiert die darauf folgende septische Phase der Erkrankung. Sie tritt typischerweise erst nach zwei bis vier Wochen auf und kennzeichnet einen schweren Verlauf der akuten Pankreatitis, der bei 40 bis $70 \%$ der Patienten eintritt (Isenmann und Beger 1999). Die Wahrscheinlichkeit der Superinfektion der Nekrosen steigt mit dem Nekroseausmaß und mit zunehmender Verlaufsdauer der Erkrankung (Beger et al. 1986). 
Wie Widdison et al. 1994 zeigen konnten, finden sich in Pankreasnekrosen überwiegend gramnegative und enterische Bakterien. Dies legt die Vermutung nahe, dass die Bakterien aufgrund der engen anatomischen Beziehung des Quercolons zum Pankreas durch Translokation in die Nekroseareale gelangen (Widdison et al. 1994). Eine neuere Studie konnte an Ratten die Beobachtung machen, dass auch aus dem Dünndarm stammende Bakterien für die bakterielle Kontamination der Nekrosen verantwortlich sind (Fritz et al. 2010).

In den letzten Jahren hat sich das in Pankreasnekrosen nachgewiesene Erregerspektrum gewandelt. Insbesondere zeigt sich eine Zunahme an grampositiven Bakterien und Pilzen, speziell CandidaSpezies. Dieses Phänomen lässt vermuten, dass ein Zusammenhang mit der routinemäßigen Gabe von Antibiotika besteht, die eine Candida-Besiedelung begünstigen, weil sie das bakterielle Milieu zugunsten der Pilze verändern. Eindeutig konnte belegt werden, dass bei positivem Candida-Nachweis die Letalität der Erkrankten erheblich steigt (Isenmann et al. 2002, Widdison et al. 1994).

Verkompliziert werden kann eine akute nekrotisierende Pankreatitis durch sekundäre Organdysfunktionen und - schäden, Arrosionen von vaskulären Strukturen und Nachbarorganen mit resultierender Hohlorganperforation. Nach erfolgter Ausheilung einer akuten Pankreatitis treten je nach Ausmaß der parenchymatösen Zerstörung des Pankreas chronische Folgezuständen auf. Diese umfassen neben strukturellen Veränderungen des Organs in Form von Verkalkungen, Pseudozysten, Pankreasfisteln und Gangstenosen insbesondere persistierende Störungen der exo- und endokrinen Funktion, entsprechend resultieren Maldigestionssyndrome beziehungsweise eine diabetische Stoffwechsellage infolge eines Insulinmangels (Connor et al. 2005, Zhou et al. 2015).

\subsubsection{Diagnose}

Die Diagnostik der akuten Pankreatitis beruht auf klinischer Untersuchung, Labordiagnostik und Bildgebung.

Leitsymptom der akuten Pankreatitis ist ein plötzlich auftretender epigastrischer Schmerz mit gürtelförmiger Ausstrahlung in den Rücken. Dieser ist häufig begleitet von Übelkeit und Erbrechen. Palpatorisch imponiert ein druckdolentes, geblähtes Abdomen "gummiartiger" Konsistenz. In seltenen Fällen werden Hautveränderungen in Form von lividen oder bräunlichen Verfärbungen periumbilikal (Cullen-Zeichen) und in der Flankenregion (Grey-Turner-Zeichen) beobachtet, die einen schweren Verlauf erwarten lassen (Runzi et al. 2000).

Labordiagnostisch bestätigt neben allgemeinen Entzündungszeichen wie einer Leukozytose die um etwa das Dreifache erhöhte pankreasspezifische Serumlipase bzw. -amylase die Diagnosestellung, 
wobei die Lipase der Amylase in Spezifität überlegen ist. Die Amylase kann dabei das 30-Fache, die Lipase das 200-Fache der oberen Referenzgrenze erreichen. Eine Lipaseerhöhung persistiert im Vergleich zur Amylase meist länger. Allerdings können aus ihrer Höhe keine Rückschlüsse auf den Schweregrad der Erkrankung gezogen werden, da es bei einer fortschreitenden Nekrose oder gar einer Totalnekrose des Pankreas zu einem Abfall des Wertes bis hin zu seinem Normwert kommen kann. Es besteht die Gefahr, dies als einen Heilungsprozess fehlzudeuten (Gumaste et al. 1993, Lankisch et al. 1999). Darüber hinaus scheint die Höhe des Serum-Gesamtkalziums und des Albumin-korrigierten Kalziums innerhalb der ersten 24 Stunden der Erkrankung zur Prognoseabschätzung hilfreich zu sein (Gutierrez-Jimenez et al. 2014). Der Laborparameter, der bei engmaschiger Verlaufskontrolle den Verdacht einer nekrotisierenden Pankreatitis begründet, ist das CRP. Persistierende oder erneut ansteigende Werte von über $15 \mathrm{mg} / \mathrm{dl}$ gelten als aussagekräftig. Des Weiteren wird momentan über die Aussagekraft des Laborparameters Procalcitonin diskutiert. Dieser wird als Akutphaseparameter für die Unterscheidung bakterieller und nicht bakterieller Entzündungen eingesetzt und erreicht rasch innerhalb von 1-2 Tagen sein Maximum. In einer retrospektiven Studie konnte eine direkte Korrelation zwischen Anstieg dieses Parameters und auftretender Nekroseinfektion beobachtet werden (Rau et al. 1997). Zu widersprüchlichen Ergebnissen kamen weitere Untersuchungen, die hervorhoben, dass es sich dabei um einen unspezifischen Parameter handelt. Dieser könne zwar zur frühen Unterscheidung zwischen leichter und schwerer Form der Pankreatitis beitragen, einen Rückschluss über den Krankheitsverlauf jedoch nicht geben (Kylanpaa-Back et al. 2001, Muller et al. 2000).

Liegt eine biliäre Ursache der akuten Pankreatitis vor, ist eine frühzeitige Identifikation der biliären Genese wichtig, um schnellstmöglich eine zielgerichtete Therapie im Sinne einer endoskopischen Steinentfernung durchführen zu können. Neben Cholestase-Parametern (AP, y-GT), Bilirubin und Transaminasen (GOT, GPT) sind Sonographie von Gallenblase und Gallenwegen unentbehrlich (Ney et al. 2005). Ein Anstieg der Transaminasen um mehr als das Dreifache hat einen positiven prädiktiven Wert von 95 \% (Tenner et al. 1994). Der sonographische Nachweis eines dilatierten Gallengangs oder einer stein- und sludgegefüllten Gallenblase wird als indirekter Nachweis einer biliären Pankreatitis gewertet. Ebenfalls konnte in zahlreichen Studien gezeigt werden, dass auch eine vorerst als idiopathisch eingestufte akute Pankreatitis in $13 \%$ bis $73 \%$ der Fälle durch eine Mikrolithiasis oder bereits abgegangene Steine hervorgerufen wurde. Ein steinfreier Gang schließt also folglich ein Gallensteinleiden als Ursache nicht aus (Garg et al. 2007).

Bildgebende Verfahren dienen der weiteren Diagnosesicherung. Während die Sonographie zur Beurteilung der Gallenwege durchaus als sinnvoll erachtet wird, ist sie zur Beurteilung des Pankreas 
aufgrund von Lufteinschlüssen nur eingeschränkt sensitiv. Bei schweren Verlaufsformen empfiehlt sich die kontrastmittelverstärkte Computertomographie (KM-CT). Bereits drei Tage nach Symptombeginn kann mittels KM-CT der Nachweis von Parenchymnekrosen des Pankreas sowie extrapankreatischen Nekrosen erfolgen (Balthazar 2002, Dervenis et al. 1999). Eine weitere bildgebende Methode zur Diagnose der akuten Pankreatitis stellt die Magnetresonanztomographie dar (Arvanitakis et al. 2004). Diese Bildgebung erhält jedoch erst nach etwa 7 Tagen ihre Aussagekraft, da das Ausmaß der Erkrankung zu früheren Zeitpunkten so gut wie nicht erfasst werden kann (Munoz-Bongrand et al. 2001).

Zur zuverlässigen Identifikation von infizierten Nekrosen steht die sonographisch- oder computertomographisch-geführte Feinnadelpunktion mit anschließender Gramfärbung des Aspirats zur Verfügung (Paye et al. 1998).

Weiterhin stehen bezüglich der Prognose der Erkrankung einige frühe Marker zur Verfügung, die prädiktive Aussagekraft besitzen, wie beispielsweise der Blutzucker. So zeigte eine im Jahr 2001 veröffentlichte Studie, dass eine Hyperglykämie eine hohe Sensitivität (28 \%) und einen hohen neg. prädiktiven Wert (92 \%) für das Auftreten von Nekrosen hat (Lankisch et al. 2001b). Ähnliches gilt für den Hämatokritwert. Lag dieser bei Patienten bei $>44 \%$, konnten hohe neg. prädiktive Werte für eine schwere Verlaufsform ermittelt werden (Brown et al. 2000).

Für die Aussage eines hohen "negativ prädiktiven Wertes“ gilt, dass nicht jeder Patient mit hohen pathologischen Werten eine Nekrose entwickelt, liegen diese Parameter jedoch im Normbereich, ist eine nekrotisierende Form höchst unwahrscheinlich.

So konnte im Umkehrschluss herausgefunden werden, dass eine frühzeitige Senkung des Hämatokrits durch Hämodilution unter einen Wert von $35 \%$ die einzige Therapieform ist, die ursächlich zur Risikominimierung einer Nekrosebildung im Pankreas führt (Brown et al. 2002).

\subsubsection{Scoring-Systeme}

Da es sich bei der akuten Pankreatitis um eine potentiell lebensbedrohliche Erkrankung handelt, ist eine frühzeitige Abschätzung des klinischen Verlaufes sehr wichtig. Hierfür wurden Scoring-Systeme entwickelt, die durch Kombination verschiedener diagnostischer Kriterien den Schweregrad der Verlaufsform, die Prognose des Patienten und spezifische Komplikationen der Pankreatitis frühzeitig anzeigen sollen und anhand derer die weiteren therapeutischen Maßnahmen abgelesen werden können. 
Der Ranson-Score dient der Beurteilung von Schweregrad der Erkrankung sowie der Abschätzung der Letalität der akuten Pankreatitis. Es basiert auf Befunden, die sowohl direkt bei Aufnahme als auch innerhalb der ersten 48 Stunden erhoben werden. Werden von diesen insgesamt 11 Kriterien 3 oder mehr erfüllt, so handelt es sich höchstwahrscheinlich um eine schwere Form der akuten Pankreatitis (Ranson 1982).

\begin{tabular}{|c|c|c|c|}
\hline \multicolumn{4}{|c|}{ Bei Aufnahme } \\
\hline \multicolumn{2}{|c|}{ Alter $>55$ Jahre } & \multicolumn{2}{|l|}{1 Punkt } \\
\hline \multicolumn{2}{|c|}{ Leukozyten $>16.000 / \mathrm{mm}^{3}$} & \multicolumn{2}{|l|}{1 Punkt } \\
\hline \multicolumn{2}{|c|}{$\mathrm{LDH}>350 \mathrm{U} / \mathrm{I}$} & \multicolumn{2}{|l|}{1 Punkt } \\
\hline \multicolumn{2}{|c|}{ ASAT (GOT) > $250 \mathrm{U} / \mathrm{l}$} & \multicolumn{2}{|l|}{1 Punkt } \\
\hline \multicolumn{2}{|c|}{ Glukose $>11,1 \mathrm{mmol} / \mathrm{l}(200 \mathrm{mg} / \mathrm{dl})$} & \multicolumn{2}{|l|}{1 Punkt } \\
\hline \multicolumn{4}{|c|}{ Innerhalb von 48 Stunden } \\
\hline \multicolumn{2}{|c|}{ Hämatokrit-Abfall um mehr als 10 \% } & \multicolumn{2}{|l|}{1 Punkt } \\
\hline \multicolumn{2}{|c|}{ Harnstoff-Anstieg über $1,8 \mathrm{mmol} / \mathrm{l}$ (> $5 \mathrm{mg} / \mathrm{dl}$ ) } & \multicolumn{2}{|l|}{1 Punkt } \\
\hline \multicolumn{2}{|c|}{ Serumkalzium $<2 \mathrm{mmol} / \mathrm{l}$} & \multicolumn{2}{|l|}{1 Punkt } \\
\hline \multicolumn{2}{|c|}{$\mathrm{PaO}_{2}<8 \mathrm{kPa}(<60 \mathrm{~mm} \mathrm{Hg})$} & \multicolumn{2}{|l|}{1 Punkt } \\
\hline \multicolumn{2}{|c|}{ Basendefizit $>4 \mathrm{mEq} / \mathrm{l}$} & \multicolumn{2}{|l|}{1 Punkt } \\
\hline \multicolumn{2}{|c|}{ Flüssigkeitsbilanz > $61 / 48 \mathrm{~h}$} & \multicolumn{2}{|l|}{1 Punkt } \\
\hline \multicolumn{2}{|c|}{ Letalität der akuten Pankreatitis } & \multicolumn{2}{|r|}{ Beurteilung } \\
\hline 0-2 Punkte: & Letalität $<5 \%$ & 0-2 Punkte: & milde Pankreatitis \\
\hline 3-4 Punkte: & Letalität 15-20 \% & $\geq 3$ Punkte: & schwere Pankreatitis \\
\hline 5-6 Punkte: & Letalität $40 \%$ & & \\
\hline > 6 Punkte: & Letalität >99 \% & & \\
\hline
\end{tabular}

Tabelle 1: Ranson-Score (Ranson 1982)

Bei einer Vergleichsstudie aller momentan existierenden Scoring-Systeme im Hinblick auf die Vorhersagegenauigkeit des Schweregrades der akuten nekrotisierenden Pankreatitis konnte gezeigt werden, dass die Bestimmung von Interleukin (IL-) 6 am ersten Tag und die CRP-Konzentration am zweiten Tag nach Aufnahme die höchste prädiktive Aussagekraft für die Früherkennung von Pankreasnekrosen aufwiesen, während die Erhebung des Ranson-Scores und APACHE-II-Scores die besten Ergebnisse zur Vorhersage der Mortalität erzielten (Khanna et al. 2013). Beide recht komplexen Scoring-Systeme haben gemeinsam, dass sie eine ausreichende Abschätzung erst nach $48 \mathrm{~h}$ 
gewährleisten können. Da sie ebenfalls recht aufwändig sind, werden sie im klinischen Alltag nicht regelhaft genutzt. Dennoch findet der Ranson-Score die häufigste Anwendung.

\subsection{Therapieverfahren}

\subsubsection{Konservative Therapie}

Wesentliche Verbesserungen in frühzeitiger Diagnosesicherung und intensivmedizinischer Therapie der akuten Pankreatitis haben dazu geführt, dass in den vergangenen Jahren eine stetige Reduzierung der Sterblichkeit erzielt werden konnte (Bank et al. 2002). Durch Optimierung von intensivmedizinischer Therapie und interdisziplinären Behandlungsstrategien konnte bei der schweren nekrotisierenden Form die Sterblichkeit von $40 \%$ auf $20 \%$ gesenkt werden (Runzi et al. 2005).

Die Behandlung erfolgt zunächst symptomatisch und richtet sich vor allem nach dem Schweregrad der Erkrankung. Da es hierfür noch an einem zuverlässigen Prädiktor mangelt, müssen alle Patienten engmaschig überwacht werden. Die Behandlung der akuten Pankreatitis erfolgt in erster Linie als konservative, medikamentös basierte Therapie. Erst bei Auftreten von schwerwiegenden Komplikationen gewinnt die Chirurgie an Bedeutung.

Beim Nachweis von Nekrosen im CT, erhöhten CRP- und Procalcitoninwerten sollte wegen des erhöhten Risikos einer bakteriellen Superinfektion mit septischen Komplikationen die Überwachung mit täglicher klinischer Untersuchung und Registrierung von Vitalparametern auf einer Intensivstation erfolgen. Hier werden konservative Maßnahmen wie Flüssigkeitssubstitution, Analgesie, ggf. Antibiotikatherapie und falls erforderlich, Organersatzverfahren durchgeführt (Banks et al. 2006, Runzi et al. 2000). Da es bei der schweren akuten Pankreatitis zu hohen Flüssigkeitsverlusten kommen kann, steht die Volumensubstitution im Vordergrund der Akutmaßnahmen. Durch regelmäßige Messung des zentralen Venendrucks, der Diurese und des Hämatokrits ist dies zu überwachen. Eine orale Nahrungskarenz bis zur Schmerzfreiheit wird empfohlen. Ein Kostaufbau kann danach mit leichtverdaulicher Nahrung begonnen werden.

Falls bei einem schweren Verlauf eine künstliche Ernährung erforderlich wird, ist die enterale der parenteralen Ernährung vorzuziehen, da hierbei das Risiko infektiöser Komplikationen, Multiorganversagen, Notwendigkeit chirurgischer Intervention und Letalität nachweislich verringert wird (Abou-Assi et al. 2002, Villatoro et al. 2010). Der Applikationsort der enteralen Ernährung ist nicht von Bedeutung. Wie eine Studie von Eatock et al. ergab, bringt die jejunale Sonde offenbar keinen Vorteil gegenüber der Magensonde (Eatock et al. 2005).

Eine suffiziente Analgesie stellt ebenfalls einen essentiellen Therapiepfeiler dar. Hierfür ist der Einsatz von Opioiden meist unumgänglich. Aufgrund des bei vielen Opiaten entstehenden Papillenspasmus 
sollten Präparate wie Buprenorphin oder Pentazocin verabreicht werden (Staritz 1988). Eine adäquate Schmerztherapie durch die parenterale Gabe des Lokalanästhetikums Procain oder auch eine Kombination aus diesem mit einem Opiat konnte nicht belegt werden (Jakobs et al. 2000, Kahl et al. 2004).

Wird eine biliäre Ursache der akuten Pankreatitis vermutet, ist eine endoskopisch retrograde Cholangiographie (ERCP) mit Papillotomie und Steinextraktion indiziert. Eine frühzeitige Steinentfernung reduziert nachweislich die Morbidität und Mortalität bei biliärer Pankreatitis (Sharma und Howden 1999). Wird die Gallenblase nach akuter Pankreatitis langfristig belassen, so kommt es in bis zu 63 \% der Fälle zu einem Rezidiv der biliären Pankreatitis. Zur Rezidivprophylaxe ist daher die Cholezystektomie idealerweise nach Ausheilung der akute Entzündung indiziert (Ando et al. 2003, Venneman et al. 2005).

Nach wie vor besteht kein allgemeiner Konsens über eine prophylaktische Antibiotikagabe bei akuter Pankreatitis. Die aktuellen Leitlinien der American Gastroenterological Association empfehlen beispielsweise eine prophylaktische Antibiotikatherapie nur bei Patienten mit einer substantiell nekrotisierenden Pankreatitis, definiert als $>30 \%$ Nekrosen im Pankreas, und diese nur für 14 Tage (Tenner et al. 2013). Empfohlene Antibiotika sind Carbapeneme oder Ciprofloxacin mit Metronidazol (Forsmark et al. 2007). Eine im Jahr 2010 veröffentlichte Übersichtsstudie zeigte, dass eine routinemäßig durchgeführte prophylaktische Antibiose generell keine Vorteile bringt, sondern vielmehr eine Selektion resistenter Keime begünstigt und die Inzidenz von Pilzinfektionen steigert (Villatoro et al. 2010). Auf der anderen Seite konnten Sharma und Howden zeigen, dass im Falle von schweren nekrotisierenden Verlaufsformen durch eine prophylaktische Antibiotikagabe eine signifikante Reduktion der Letalität um 12,3 \% und der septischen Komplikationen um 21,1 \% erzielt werden kann (Sharma und Howden 2001). Da die sekundäre Infektion der Nekrosen die Ursache für die hohe Mortalität der akuten Pankreatitis darstellt, kommt einer frühzeitigen Erkennung und adäquaten Therapie große Bedeutung zu. Ein gemeinsamer Konsens hierzu mit evidenzbasierenden Daten konnte noch nicht gefunden werden. Europäische Leitlinien zu diesem Thema stehen noch aus.

Da die Dysfunktion der Darmbarriere sowie die Translokation von Darmbakterien maßgeblich für die Superinfektion der Nekrosen und der daraus resultierenden hohen Mortalität der Erkrankung verantwortlich gemacht werden, wurden in den letzten Jahren große Erwartungen hinsichtlich des gesundheitsfördernden Effektes von Probiotika geweckt (Guarner und Malagelada 2003). So könnten diese als probiotisch bezeichneten Bakterienstämme infektiöse Komplikationen minimieren, indem sie die bakterielle Fehlbesiedelung des Dünndarms verhindern. Ziel hierbei ist es, die mukosale Barrierefunktion zu erhalten und eine Entzündungsreaktion zu dämpfen (Bengmark 1998, Guarner und 
Malagelada 2003). Eine multizentrisch durchgeführte Studie von Besselink et al. dazu widerlegte jedoch diese Annahme und zeigte, dass die prophylaktische Gabe probiotischer Präparate mit einer höheren Letalität der Erkrankten assoziiert ist (Besselink et al. 2008). Daher wird der Einsatz von Probiotika zur Therapie beziehungsweise zur Prophylaxe von Superinfektionen der Pankreasnekrosen nicht empfohlen.

\subsubsection{Stellenwert der Chirurgie in der Behandlung der akuten nekrotisierenden Pankreatitis}

In den letzten Jahren hat ein Paradigmenwechsel in der Behandlungsstrategie der akuten nekrotisierenden Form der Pankreatitis stattgefunden. Das Konzept der frühzeitigen chirurgischen Intervention wurde durch eine primär konservative Stabilisierung ersetzt (Bausch et al. 2012). Ebenso stellen nicht infizierte Nekrosen heutzutage keine Operationsindikation mehr da (Uhl et al. 2002).

Die Indikation zur chirurgischen Intervention ist gegeben, wenn eine biliare Genese eine Cholezystektomie erforderlich macht, infizierte Pankreasnekrosen interventionell nicht zu sanieren sind oder andere Komplikationen wie Blutungen oder Hohlorgan-Perforationen auftreten (Besselink et al. 2013).

\subsubsection{Chirurgische Therapie zur Behandlung der Ursachen der Pankreatitis}

Bei akuter Pankreatitis auf dem Boden einer Cholezystolithiasis ist die chirurgische Therapie im Sinne einer Cholezystektomie indiziert, da ein langfristiges Belassen der Gallenblase mit einer Rezidivwahrscheinlichkeit von bis zu 63 \% einhergeht (Venneman et al. 2005). Der optimale Zeitpunkt wird abhängig gemacht von dem Schweregrad der Pankreatitis, von biliären Komplikationen und von der Notwendigkeit bei schwerem Verlauf eventuell andere chirurgische Interventionen durchführen zu müssen (Nealon et al. 2004, Uhl et al. 2002).

Grundsätzlich gilt, dass eine chirurgische Sanierung erst in einem entzündungsfreien Intervall erfolgen sollte. Ist bei einer milden Form der Pankreatitis der Stein nicht bereits spontan abgegangen, sollte frühelektiv, das heißt innerhalb der ersten $<24 \mathrm{~h}$ nach Krankheitsbeginn, eine ERCP mit Papillotomie und Steinextraktion erfolgen. Eine laparoskopische Cholezystektomie sollte noch während desselben stationären Aufenthalts innerhalb einer Woche durchgeführt werden (Kimura et al. 2006). Sind biliäre Komplikationen nachweisbar, stehen eine Notfall-ERCP und Steinextraktion im Vordergrund. Patienten ohne Hinweis auf Komplikationen profitieren nach einer Metaanalyse von sieben kontrolliert randomisierten Studien von einer sofortigen ERCP nicht (Petrov et al. 2008). 


\subsubsection{Chirurgische Therapie bei akuter nekrotisierender Pankreatitis ohne Kolektomie}

Der alleinige Nachweis von Nekrosen stellt heutzutage keine Operationsindikation mehr dar. Bei Nachweis von Superinfektionen gilt das chirurgische Débridement im Sinne einer Nekrosektomie als Goldstandard (Isaji et al. 2006). Dabei ist der Zeitpunkt der chirurgischen Intervention bedeutsam. Sowohl ältere als auch neuere Beobachtungsstudien zeigen, dass die chirurgische Intervention, wenn möglich, erst mindestens vier Wochen nach Krankheitsbeginn erfolgen sollte. Vorteil eines späteren chirurgischen Eingriffs ist die zunehmende Demarkierung der nekrotischen Areale, was die Abgrenzung zu vitalem Gewebe erleichtert, das Blutungsrisiko reduziert und somit den maximalen Erhalt funktionellen Gewebes sicherstellt. Frühe Eingriffe gehen häufiger mit Komplikationen und langfristigen Schäden, wie beispielsweise der Pankreasinsuffizienz sowie einer erhöhten Sterblichkeitsrate einher (Bausch et al. 2012, Mier et al. 1997).

Ziel der chirurgischen Therapie ist es, das superinfizierte avitale Gewebe zu entfernen. Die Bursa Omentalis wird eröffnet. Parenchymschonend werden nekrotische Areale mit der Hand oder der Pinzette entfernt. Anschließend wird eine ausgiebige Lavage aller Exudatstraßen durchgeführt. Da es sich häufig um fortschreitende Prozesse handelt, sind in vielen Fällen mehrmalige Relaparotomien nötig (Amano et al. 2010).

Um postoperativ ein weiteres Spülen des Abdomens zur Entfernung infektiösen Sekrets zu gewährleisten, existieren mehrere Verfahren, die mit ähnlichen Ergebnissen durchgeführt werden.

Das offene Verfahren kann entweder als open packing oder als Etappenlavage erfolgen, das geschlossene Verfahren als closed packing und Bursalavage. Das Prinzip des offenen Verfahrens besteht aus mehreren Revisions-Operationen: bei der Etappenlavage wird alle 24-72 $\mathrm{h}$ eine Relaparotomie durchgeführt, beim open packing wird das Abdomen dabei zwischenzeitlich nicht verschlossen (Werner et al. 2003). Neben Bauchtüchern werden auch Vakuumschwämme zur Säuberung und Granulationsförderung verwendet. Das geschlossene Verfahren sieht eine einzeitige Operation vor, bei der eingelegte Drainagen postoperativ zu einer kontinuierlichen Ableitung von Debris und Sekret führen sollen (closed packing) (Fernandez-del Castillo et al. 1998). Bei der Bursalavage wird zusätzlich für Tage bis Wochen kontinuierlich über in die Bursa Omentalis und die ehemaligen Nekrosestraßen eingebrachte Spülkatheter eine Dauerspülung der Bursa durchgeführt (Beger et al. 1988). Sofern die Techniken von erfahrenen Chirurgen durchgeführt werden, konnten für jedes Verfahren Mortalitätsraten von unter 10-20 \% erreicht werden (Werner et al. 2005b). Beiden offenen Verfahren ist nachteilig, dass sie mit vielen postoperativen Komplikationen wie gastrointestinalen Fisteln, Magenausgangsstenosen, Narbenhernien und lokalen Blutungen einhergehen (Werner et al. 2005a). 
Um das operative Trauma und die durch die wiederholten Laparotomien bedingten Komplikationen und Folgezustände zu vermeiden, sind minimalinvasive Therapieoptionen derzeit Gegenstand vieler Studien. Eine systematische Evaluation steht jedoch noch aus. Eine niederländische Arbeitsgruppe hatte bereits 2010 in einer Studie an 88 Patienten zeigen können, dass eine Step-up-Therapie aus Drainage über einen perkutanen Katheter mit folgender minimal-invasiver Nekrosektomie verglichen zum offenen, chirurgischen Vorgehen mit einer signifikant geringeren Komplikationsrate assoziiert ist (55 \% zu 81 \%) (van Santvoort et al. 2010). Eine weitere Studie derselben Arbeitsgruppe, in der die endoskopisch-transgastrale Nekrosektomie mit der chirurgischen offenen Nekrosektomie verglichen wurde, konnte dieses Ergebnis unterstreichen und erheblich weniger postoperative Entzündungsreaktionen wie auch Spätkomplikationen beobachten (Bakker et al. 2012). Valide Daten größerer Studien sowie Metaanalysen sind jedoch momentan noch nicht verfügbar. Da es bisher keine generellen Empfehlungen zur Durchführung minimalinvasiver Therapieoptionen gibt, bleibt dieser Therapieansatz spezialisierten Zentren vorbehalten.

Als Komplikationen nach Nekrosektomie werden sowohl exokrine als auch endokrine Insuffizienzen des Pankreas wie auch Gallengangsstenosen, Fisteln, Pankreasabszesse oder - pseudozysten, die sich durchschnittlich nach 29 Monaten postoperativ bilden, beschrieben (Connor et al. 2005).

\subsubsection{Konzept der kombinierten Nekrosektomie und Kolektomie}

Kolonnekrosen und - perforationen sind als Komplikation der akuten nekrotisierenden Pankreatitis mit einer hohen Mortalität assoziiert (Kriwanek et al. 1999). Eine Studie von van Minnen et al. ermittelte eine Inzidenz von das Kolon betreffenden Komplikationen von $15 \%$ in Zusammenhang mit schweren akuten Pankreatitiden (Van Minnen et al. 2004).

Eine Übersichtsstudie des Zeitraumes 1964-1992 von Adams et al. konnte einen Unterschied in Charakter und Schweregrad der Kolonkomplikation bei Pankreatitis zeigen. Während bei einer chronischen Pankreatitis pankreato-kolische Fisteln durch lokale Exzision und segmentale Resektion des Dickdarms gut therapierbar waren, hatte bei der akuten nekrotisierenden Pankreatitis das Überschreiten des nekrotisierenden Entzündungsprozesses auf den Dickdarm schwerwiegendere Folgen für die Patienten. Trotz einer aggressiven chirurgischen Therapie in Form einer Kolektomie zur Sanierung des arrodierten Kolons war die Einbeziehung des Kolons in den Entzündungsprozess durch Sepsis und Multiorganversagen mit einer hohen Mortalität assoziiert (Adams et al. 1994).

Die Pathomechanismen dieses Prozesses sind derweil weiterhin Gegenstand vieler Diskussionen. Die führende Theorie geht davon aus, dass es während der akuten nekrotisierenden Pankreatitis zu einer direkten Ausbreitung der Pankreasenzyme durch das Peritoneum in Richtung des Mesokolons und 
Kolons kommt. Eine andere Theorie macht eine Thrombose oder Kompression einer der Mesenterialarterien und - venen mit nachfolgender Thrombose für die Ischämie und Kongestion des Kolonabschnitts mit nachfolgender Barrierestörung und bakterieller Translokation verantwortlich. Eine dritte Hypothese besagt, dass die Hypotonie und Zentralisation des Blutes, ausgelöst durch den septischen Schock, zu einer Minderperfusion des Kolons und sekundär zu einer ischämisch bedingten Perforation des betroffenen Kolonabschnittes führt.

Letztlich ist bei allen hypothetischen Prozessen die Mukosa nicht mehr in der Lage, die Barrierefunktion aufrechtzuerhalten. Es kommt zu einer Translokation von Bakterien und Toxinen aus dem Darmlumen mit daraus resultierender Kontamination der Pankreasnekrosen mit enterischen Bakterien und der Ausbildung einer Sepsis (Doberneck 1989, Kriwanek et al. 1994, Kriwanek et al. 1996).

Daher muss bei klinischen Hinweisen auf eine Superinfektion der Nekrosen an eine Beteiligung des Kolons am lokal entzündlichen Prozess gedacht werden (Russell et al. 1983).

Bei Nachweis einer segmentalen Ischämie des Kolons oder einer Perforation muss zur kausalen Therapie das betroffene Darmsegment reseziert werden (Van Minnen et al. 2004).

\subsection{Zielsetzung der Arbeit}

Ausgangspunkt der Untersuchungen war die Beobachtung, dass über einen vergleichbaren Zeitraum die chirurgische Therapie der akuten nekrotisierenden Pankreatitis an zwei verschiedenen Universitätskliniken unterschiedlich konzeptioniert war.

Basierend auf dieser Beobachtung war Fragestellung der vorliegenden Arbeit, inwieweit Patienten mit komplizierter akuter nekrotisierender Pankreatitis von einer ausgedehnten chirurgischen Therapie mit der Therapieoption der Kolektomie zur Fokussanierung profitieren.

In der aktuellen Studie wurde deshalb anhand retrospektiv erhobener Daten das chirurgische Konzept zur Therapie der akuten nekrotisierenden Pankreatitis bestehend aus kombinierter Nekrosektomie und Kolektomie analysiert und mit der Therapie der alleinigen Nekrosektomie verglichen. 


\section{$2 \quad$ Patienten und Methoden}

\subsection{Patientenkollektiv}

In der aktuellen Studie wurde anhand retrospektiv erhobener Daten das chirurgische Konzept zur Therapie der akuten nekrotisierenden Pankreatitis, bestehend aus kombinierter Nekrosektomie und Kolektomie, analysiert und mit der Therapie der alleinigen Nekrosektomie verglichen. Hierzu erfolgte der Vergleich von zwei Patientenkollektiven aus zwei deutschen chirurgischen universitären Zentren. In der Klinik für Allgemein-, Viszeral, Kinder- und Gefäßchirurgie des Universitätsklinikums des Saarlandes in Homburg/Saar war das Konzept der Kolektomie in das chirurgische Management der nekrotisierenden Pankreatitis implementiert. In der Klinik für Allgemein-, Viszeral- und Kinderchirurgie der Universitätsmedizin Göttingen war dieses zusätzliche chirurgische Verfahren bei Patienten mit nekrotisierender Pankreatitis nicht Teil der Behandlung.

Aus dem jeweiligen Klinik-Informationssystem wurden anhand der ICD-10-Codierung die Patienten selektioniert, bei denen der Diagnoseschlüssel K85 hinterlegt war. Die Homburger Daten umfassten Patienten, die im Zeitraum von Januar 2002 bis März 2012 aufgrund einer akuten nekrotisierenden Pankreatitis behandelt wurden. Der Auswertungszeitraum in Göttingen erstreckte sich von Januar 2008 bis Dezember 2012.

Von sämtlichen Patienten wurden nachfolgend die elektronischen und papiergebundenen Patientenakten eingesehen und die zuvor definierten Kennzahlen erhoben.

Anhand der ICD-10-Codierung wurden alle diejenigen Patienten in die Studie eingeschlossen, die an einer komplizierten akuten nekrotisierenden Pankreatitis litten. Dabei wurde die Notwendigkeit einer intensivmedizinischen Behandlung, sowie einer chirurgischen Intervention im Rahmen des stationären Aufenthaltes als Voraussetzung für den Einschluss in die Studie angesehen. Unter Verwendung dieser Kriterien ergab sich ein Kollektiv von 72 Patienten, 38 Patienten aus Homburg/Saar und 34 Patienten aus Göttingen. Im Rahmen der weiteren Stratefizierung wurden aus diesem Patientenkollektiv die Patienten mit schwerem Verlauf für die weitere Auswertung herangezogen. Hierfür wurde die Katecholaminpflichtigkeit als Zeichen der Kreislaufinsuffizienz im Rahmen des Aufenthalts auf Intensivstation als ein weitergehendes Einschlusskriterium festgelegt. Schlussendlich konnte so ein Kollektiv von 56 vergleichbaren Patienten (37 Patienten aus Homburg/Saar und 19 Patienten aus Göttingen) in die Analyse einbezogen werden.

Das Ethikvotum für die vorliegende retrospektive Analyse wurde am 02.10.2012 erteilt (7/10/12An). 


\subsection{Datenerhebung / Methoden}

Zur Erhebung der Daten wurden aus den zur Verfügung stehenden Patientenakten festgelegte Parameter erfasst und in einer Microsoft Excel-basierten Tabelle zusammengestellt.

Diese Daten umfassten demographische Daten (Alter und Geschlecht), Angaben zur Ätiologie der Pankreatitis sowie bestehende Komorbiditäten, perioperative Daten (OP-Dauer, Anzahl der OPs, chirurgische Prozeduren), Krankenhausverweildauer, Aufenthaltsdauer auf Intensivstation, Mortalität sowie sekundäre Organkomplikationen und Notwendigkeit von Organersatzverfahren.

Nach dem Operationen- und Prozedurenschlüssel (IKPM) wurden alle für die akute nekrotisierende Pankreatitis relevanten chirurgischen Maßnahmen entschlüsselt.

\begin{tabular}{|c|l|}
\hline IKPM & \\
\hline $\mathbf{5 - 5 2 1}$ & Lokale Exzision und Destruktion von erkranktem Gewebe des Pankreas \\
\hline $\mathbf{5 - 5 2 3}$ & Innere Drainage des Pankreas \\
\hline $\mathbf{5 - 5 2 4}$ & Partielle Resektion des Pankreas \\
\hline $\mathbf{5 - 5 2 5}$ & (Totale) Pankreatektomie \\
\hline $\mathbf{5 - 4 5 5}$ & Partielle Resektion des Dickdarms \\
\hline $\mathbf{5 - 4 5 6}$ & (Totale) Kolektomie \\
\hline
\end{tabular}

Tabelle 2: IKPM der akuten nekrotisierenden Pankreatitis

\subsubsection{Primäre Endpunkte}

Um den Erfolg der zusätzlichen chirurgischen Maßnahme der Kolektomie objektiv bewerten zu können, wurden als primäre Endpunkte dieser Arbeit die Krankenhausmortalität und die 30- und 90-TagesMortalität der Patienten definiert.

\subsubsection{Sekundäre Endpunkte}

Zu den sekundären Endpunkten gehörten perioperative Daten wie Verweildauer sowohl auf der Normalstation als auch auf der Intensivstation, Hospitalisationstage und Intensivtage vor der ersten OP. Zudem wurden perioperative Daten wie OP-Dauer und Anzahl der Operationen analysiert. 


\subsection{Statistische Analyse}

Die Datenanalyse erfolgte mit Hilfe des Statistikpakets SPSS (Version 20, IBM Corp.). Für die deskriptive Darstellung der Ergebnisse wurden absolute und relative Häufigkeiten, Mittelwerte, Standardabweichungen berechnet. Mittelwertunterschiede von parametrischen Daten zwischen zwei Gruppen wurden mittels t-Test beziehungsweise Mann-Whitney-U-Test auf Signifikanz geprüft, Mittelwertunterschiede zwischen mehreren Gruppen wurden mittels One-Way ANOVA mit post-hoc Vergleich nach Bonferroni geprüft. Für die Feststellung von Verteilungsunterschieden wurden der exakte Test nach Fisher beziehungsweise Chi-Quadrat-Test nach Pearson angewandt. Für eine Irrtumswahrscheillichkeit $p<0,05$ wurde eine statistische Signifikanz angenommen. 


\section{$3 \quad$ Ergebnisse}

\subsection{Patientenkollektiv}

\subsubsection{Vergleich der beiden Zentren}

Unter der Verwendung der Einschlusskriterien wie der Notwendigkeit einer intensivmedizinischen Behandlung sowie einer chirurgischen Intervention im Rahmen des stationären Aufenthaltes entstand ein primäres Kollektiv von 72 Patienten, 38 Patienten aus Homburg/Saar und 34 Patienten aus Göttingen.

Im Rahmen der weiteren Stratefizierung wurden aus diesem Patientenkollektiv die Patienten mit schwerem komplizierten Verlauf für die weitere statistische Auswertung herangezogen. Hierfür wurde die Katecholaminpflichtigkeit als Zeichen der Kreislaufinsuffizienz im Rahmen des Aufenthalts auf Intensivstation als ein weitergehendes Einschlusskriterium festgelegt. Sodass schlussendlich ein Kollektiv von 56 vergleichbaren Patienten (37 Patienten aus Homburg/Saar und 19 Patienten aus Göttingen) in die Analyse einbezogen wurden.

Da die dieser Auswertung zugrunde liegenden Daten an zwei verschiedenen universitären Zentren erhoben wurden, erfolgte ein statistischer Vergleich der Patientenkollektive aus Homburg/Saar und Göttingen, um etwaige Unterschiede in den Kollektiven zu detektieren. 


\begin{tabular}{|c|c|c|c|c|c|}
\hline & & Gesamtkollektiv & HOM & GÖ & p (HOM vs. GÖ) \\
\hline \multicolumn{2}{|l|}{$\mathrm{n}$} & 56 & 37 & 19 & \\
\hline \multicolumn{2}{|l|}{ Alter [Jahre] } & $57,2 \pm 15,0$ & $56,3 \pm 14,7$ & $59,1 \pm 15,6$ & 0,507 \\
\hline \multirow{2}{*}{ männlich } & $n$ & 32 & 22 & 10 & \multirow{4}{*}{0,777} \\
\hline & $\%$ & 57,1 & 59,5 & 52,6 & \\
\hline \multirow{2}{*}{ weiblich } & $\mathrm{n}$ & 24 & 15 & 9 & \\
\hline & $\%$ & 42,9 & 40,5 & 47,4 & \\
\hline \multirow{2}{*}{ Hyperglykämie } & $n$ & 3 & 1 & 2 & \multirow{2}{*}{1,000} \\
\hline & $\%$ & 8,8 & 6,7 & 10,5 & \\
\hline \multirow{2}{*}{ Diabetes } & $\mathrm{n}$ & 11 & 5 & 6 & \multirow{2}{*}{1,000} \\
\hline & $\%$ & 32,4 & 33,3 & 31,4 & \\
\hline \multirow{2}{*}{ Hypertonie } & $\mathrm{n}$ & 20 & 8 & 12 & \multirow{2}{*}{0,728} \\
\hline & $\%$ & 58,8 & 53,3 & 63,2 & \\
\hline \multirow{2}{*}{ Nikotinabusus } & $\mathrm{n}$ & 8 & 3 & 5 & \multirow{2}{*}{1,000} \\
\hline & $\%$ & 23,5 & 20,0 & 26,3 & \\
\hline \multirow{2}{*}{ Alkoholabusus } & $n$ & 7 & 2 & 5 & \multirow{2}{*}{0,415} \\
\hline & $\%$ & 20,0 & 12,5 & 26,3 & \\
\hline \multirow{2}{*}{ Adipositas } & $n$ & 11 & 6 & 5 & \multirow{2}{*}{0,475} \\
\hline & $\%$ & 32,4 & 40,0 & 26,3 & \\
\hline \multirow{2}{*}{ Hyperlipidämie } & $\mathrm{n}$ & 5 & 1 & 4 & \multirow{2}{*}{0,355} \\
\hline & $\%$ & 14,7 & 6,7 & 21,1 & \\
\hline
\end{tabular}

Tabelle 3: Demographische Daten und Begleiterkrankungen des Gesamtkollektivs sowie der Teilkollektive aus Homburg/Saar (HOM) und Göttingen (GÖ). Daten werden als Mittelwerte \pm SD, absolute und relative Häufigkeiten dargestellt.

Die demographischen Daten und Komorbiditäten werden in Tabelle 3 zusammengefasst.

Die statistische Auswertung zeigte keine signifikanten Unterschiede der Teilkollektive hinsichtlich der demographischen Daten und Komorbiditäten. 
Ebenso zeigte die Auswertung der der akuten nekrotisierenden Pankreatitis ätiologisch zugrunde liegenden Faktoren keine maßgeblichen Unterschiede zwischen den beiden Teilkollektiven (Tabelle 4). Signifikant unterschiedlich war einzig die Häufigkeit der Diagnose einer Rezidiv-Pankreatitis. Diese wurde in Homburg weitaus seltener gestellt als in Göttingen (5,4\% vs. $26,3 \%$; $p=0,038)$.

\begin{tabular}{|c|c|c|c|c|c|}
\hline & & Gesamtkollektiv & HOM & GÖ & p (HOM vs. GÖ) \\
\hline \multirow{2}{*}{ Cholezystolithiasis } & $\mathrm{n}$ & 26 & 7 & 9 & \multirow{2}{*}{1,000} \\
\hline & $\%$ & 47,1 & 46,7 & 47,4 & \\
\hline \multirow{2}{*}{ Alkoholabusus } & $\mathrm{n}$ & 7 & 2 & 5 & \multirow{2}{*}{0,415} \\
\hline & $\%$ & 20,0 & 12,5 & 26,3 & \\
\hline \multirow{2}{*}{ post-ERCP } & $\mathrm{n}$ & 9 & 2 & 7 & \multirow{2}{*}{0,240} \\
\hline & $\%$ & 26,5 & 13,3 & 36,8 & \\
\hline \multirow{2}{*}{ Hyperlipidämie } & $\mathrm{n}$ & 5 & 1 & 4 & \multirow{2}{*}{0,355} \\
\hline & $\%$ & 14,7 & 6,7 & 21,1 & \\
\hline \multirow{2}{*}{ Hyperglykämie } & $\mathrm{n}$ & 3 & 1 & 2 & \multirow{2}{*}{1,000} \\
\hline & $\%$ & 8,8 & 6,7 & 10,5 & \\
\hline \multirow{2}{*}{ idiopathisch } & $\mathrm{n}$ & 3 & 0 & 3 & \multirow{2}{*}{0,238} \\
\hline & $\%$ & 8,8 & 0,0 & 15,8 & \\
\hline \multirow{2}{*}{ Rezidiv-Pankreatitis } & $\mathrm{n}$ & 7 & 2 & 5 & \multirow{2}{*}{0,038} \\
\hline & $\%$ & 12,5 & 5,4 & 26,3 & \\
\hline
\end{tabular}

Tabelle 4: Ätiologie der Pankreatitis des Gesamtkollektivs sowie der Teilkollektive aus Homburg/Saar (HOM) und Göttingen (GÖ). Daten werden als absolute und relative Häufigkeiten dargestellt. 
Eine Übersicht über die Komplikationen und Organersatzverfahren ist in Tabelle 5 dargestellt.

Statistisch signifikant unterschied sich nur die Häufigkeit einer Pneumonie/ARDS. Diese wurde in Göttingen mit 57,9 \% sehr viel häufiger diagnostiziert als in Homburg mit 20,0\% ( $p=0,038)$.

\begin{tabular}{|c|c|c|c|c|c|}
\hline & & Gesamtkollektiv & Hом & GÖ & p (HOM vs. GÖ) \\
\hline \multirow{2}{*}{ Pneumonie/ARDS } & $\mathrm{n}$ & 14 & 3 & 11 & \multirow{2}{*}{0,038} \\
\hline & $\%$ & 41,2 & 20,0 & 57,9 & \\
\hline \multirow{2}{*}{ Beatmung } & $\mathrm{n}$ & 32 & 15 & 17 & \multirow{2}{*}{0,492} \\
\hline & $\%$ & 94,1 & 100,0 & 89,5 & \\
\hline \multirow{2}{*}{ akutes Nierenversagen } & $\mathrm{n}$ & 21 & 8 & 13 & \multirow{2}{*}{0,300} \\
\hline & $\%$ & 63,6 & 53,3 & 72,2 & \\
\hline \multirow{2}{*}{ Dialyse } & $\mathrm{n}$ & 19 & 9 & 10 & \multirow{2}{*}{1,000} \\
\hline & $\%$ & 55,9 & 56,3 & 55,6 & \\
\hline \multirow{2}{*}{ Leberversagen } & $\mathrm{n}$ & 7 & 1 & 6 & \multirow{2}{*}{0,104} \\
\hline & $\%$ & 20,6 & 6,7 & 31,6 & \\
\hline \multirow{2}{*}{$\begin{array}{l}\text { disseminierte intravasale } \\
\text { Koagulopathie }\end{array}$} & $n$ & 4 & 0 & 4 & \multirow{2}{*}{0,113} \\
\hline & $\%$ & 11,8 & 0,0 & 21,1 & \\
\hline \multirow{2}{*}{ Transfusionsbedarf } & $\mathrm{n}$ & 32 & 13 & 19 & \multirow{2}{*}{0,187} \\
\hline & $\%$ & 94,1 & 86,7 & 100,0 & \\
\hline
\end{tabular}

Tabelle 5: Komplikationen und Organersatztherapie des Gesamtkollektivs sowie der Teilkollektive aus Homburg/Saar (HOM) und Göttingen (GÖ). Daten werden als absolute und relative Häufigkeiten dargestellt.

Die chirurgischen Therapiemaßnahmen, die aufgrund der akuten nekrotisierenden Pankreatitis durchgeführt wurden, sind in Tabelle 6 dargestellt. Die Auswertung zeigte, dass die Homburger Patienten signifikant häufiger kolektomiert wurden $(24,3 \%, p=0,021)$, während dieses Verfahren in der Universitätsmedizin Göttingen bei keinem der Patienten durchgeführt wurde.

\begin{tabular}{|c|c|c|c|c|c|}
\hline & & Gesamtkollektiv & HOM & GÖ & p (HOM vs. GÖ) \\
\hline \multirow{2}{*}{ Kein Koloneingriff } & $\mathrm{n}$ & 39 & 24 & 15 & \multirow{2}{*}{0,364} \\
\hline & $\%$ & 69,6 & 64,9 & 78,9 & \\
\hline \multirow{2}{*}{ Kolektomie } & $\mathrm{n}$ & 9 & 9 & 0 & \multirow{2}{*}{0,021} \\
\hline & $\%$ & 16,1 & 24,3 & 0,0 & \\
\hline \multirow{2}{*}{ Kolonteilresektion } & $\mathrm{n}$ & 8 & 4 & 4 & \multirow{2}{*}{0,423} \\
\hline & $\%$ & 14,3 & 10,8 & 21,1 & \\
\hline \multirow{2}{*}{ Kolektomie bei Ersteingriff } & $n$ & 5 & 5 & 0 & \multirow{2}{*}{0,000} \\
\hline & $\%$ & 8,9 & 13,5 & 0,0 & \\
\hline
\end{tabular}

Tabelle 6: Operative Therapiemaßnahmen des Gesamtkollektivs sowie der Teilkollektive aus Homburg/Saar (HOM) und Göttingen (GÖ). Daten werden als absolute und relative Häufigkeiten dargestellt. 
Die Analyse der perioperativen Daten zeigte, dass pro Patient in Homburg mehr operative Eingriffe benötigt wurden als in Göttingen (Tabelle 7). Während in Homburg jeder Patient im Durchschnitt 6,5 mal operiert wurde, war dies in Göttingen durchschnittlich 4,9 mal der Fall.

Die Dauer der Operationen war in Homburg ebenfalls höher als in Göttingen (HOM: 122,5 min vs. GÖ: $93,7 \min ; p=0,138)$.

Statistisch signifikante Unterschiede konnten jedoch nicht gezeigt werden.

\begin{tabular}{|l|c|c|c|c|}
\hline & Gesamtkollektiv & HOM & GÖ & P (HOM vs. GÖ) \\
\hline Anzahl OPs [ $\mathrm{n}]$ & $6,0 \pm 6,5$ & $6,5 \pm 7,4$ & $4,9 \pm 4,6$ & 0,368 \\
\hline OP-Dauer [min] & $112,7 \pm 66,8$ & $122,5 \pm 57,0$ & $93,7 \pm 81,1$ & 0,138 \\
\hline OP-Nr. der Kolektomie & $2,1 \pm 1,5$ & $2,1 \pm 1,5$ & - & - \\
\hline OP-Nr. der Kolektomie nach Diagnosestellung & $1,7 \pm 1,3$ & $1,7 \pm 1,3$ & - & - \\
\hline Dauer von Diagnosestellung bis Kolektomie [d] & $15,1 \pm 16,6$ & $15,1 \pm 16,6$ & - & - \\
\hline Hospitalisation vor Kolektomie [d] & $18,2 \pm 17,1$ & $18,2 \pm 17,1$ & - & - \\
\hline Hospitalisation vor Kolonteilresektion [d] & $1,7 \pm 1,4$ & $2,0 \pm 2,8$ & $1,5 \pm 0,6$ & 0,720 \\
\hline
\end{tabular}

Tabelle 7: Perioperative Daten des Gesamtkollektivs sowie der Teilkollektive aus Homburg/Saar (HOM) und Göttingen (GÖ). Daten werden als Mittelwerte \pm SD dargestellt. 


\subsubsection{Primäre Endpunkte}

Die Analyse der primären Endpunkte zeigte keine statistisch signifikanten Unterschiede zwischen den beiden Universitätskliniken (Tabelle 8).

Die Krankenhaussterblichkeit betrug im Gesamtkollektiv 50,0\%. In Homburg verstarben 48,6 \% und in Göttingen 52,6\% $(p=1,000)$ der Patienten.

Die 30-Tages-Mortalität als weiterer primärer Endpunkt betrug im Gesamtkollektiv 21,4\% und war ebenfalls im Vergleich der beiden Zentren nicht signifikant unterschiedlich (HOM: 18,9 \%, GÖ: 26,3 \%; $p=0,516$ ). Die 90-Tages-Mortalität des Gesamtkollektivs betrug 35,7\% und zeigte ebenso bei Vergleich der beiden Zentren keine Signifikanz (HOM: 35,1\%, GÖ: 36,8 \%; p=1,000).

\begin{tabular}{|c|c|c|c|c|c|}
\hline & & Gesamtkollektiv & ном & GÖ & p (HOM vs. GÖ) \\
\hline \multirow{2}{*}{ Krankenhausmortalität } & $n$ & 28 & 18 & 10 & \multirow{2}{*}{1,000} \\
\hline & $\%$ & 50,0 & 48,6 & 52,6 & \\
\hline \multirow{2}{*}{ 30-Tages-Mortalität } & $\mathrm{n}$ & 12 & 7 & 5 & \multirow{2}{*}{0,516} \\
\hline & $\%$ & 21,4 & 18,9 & 26,3 & \\
\hline \multirow{2}{*}{ 90-Tages-Mortalität } & $n$ & 20 & 13 & 7 & \multirow{2}{*}{1,000} \\
\hline & $\%$ & 35,7 & 35,1 & 36,8 & \\
\hline
\end{tabular}

Tabelle 8: Primäre Endpunkte: Krankenhausmortalität sowie 30- und 90-Tages-Mortalitäten des Gesamtkollektivs sowie der Teilkollektive aus Homburg/Saar (HOM) und Göttingen (GÖ). Daten werden als absolute und relative Häufigkeiten dargestellt.

\subsubsection{Sekundäre Endpunkte}

Die statistische Auswertung der sekundären Endpunkte zeigte, dass die Krankenhausverweildauer insgesamt in Homburg erheblich höher lag als in Göttingen (HOM: 74,7 Tage, GÖ: 62,5 Tage; p=0,455). Gleichzeitig lag die Überlebensdauer der Verstorbenen in Homburg ebenfalls höher als in Göttingen (HOM: 73,5 Tage, GÖ: 55,1 Tage; $p=0,512$ ). Signifikante Unterschiede bei Vergleich der beiden Zentren konnten jedoch nicht nachgewiesen werden (Tabelle 9).

\begin{tabular}{|l|c|c|c|c|}
\hline & Gesamtkollektiv & HOM & GÖ & p (HOM vs. GÖ) \\
\hline Krankenhausverweildauer insgesamt [d] & $70,5 \pm 57,0$ & $74,7 \pm 60,7$ & $62,5 \pm 49,7$ & 0,455 \\
\hline Krankenhausverweildauer der Überlebenden [d] & $74,2 \pm 41,4$ & $75,9 \pm 41,3$ & $70,7 \pm 43,8$ & 0,764 \\
\hline Krankenhausverweildauer der Verstorbenen [d] & $66,9 \pm 69,4$ & $73,5 \pm 76,7$ & $55,1 \pm 55,8$ & 0,512 \\
\hline Intensivverweildauer [d] & $46,8 \pm 46,0$ & $49,6 \pm 47,9$ & $41,5 \pm 43,0$ & 0,537 \\
\hline Krankenhausverweildauer vor 1.OP [d] & $12,2 \pm 18,6$ & $13,4 \pm 21,1$ & $9,7 \pm 12,7$ & 0,487 \\
\hline Intensivverweildauer vor 1.OP [d] & $5,4 \pm 11,1$ & $6,1 \pm 12,2$ & $4,1 \pm 8,6$ & 0,512 \\
\hline
\end{tabular}

Tabelle 9: Sekundäre Endpunkte des Gesamtkollektivs sowie der Teilkollektive aus Homburg/Saar (HOM) und Göttingen (GÖ). Daten werden als Mittelwerte \pm SD dargestellt. 


\subsubsection{Subgruppenvergleich der unterschiedlichen Therapieoptionen}

Nach erfolgtem Ausschluss schwerwiegender Unterschiede in den Teilkollektiven aus Homburg und Göttingen wurden diese zu einem Gesamtkollektiv zusammengefasst. Innerhalb dieses Kollektivs wurden nachfolgend mehrere Subgruppenanalysen vorgenommen.

\subsubsection{Einfluss der verschiedenen chirurgischen Therapiekonzepte}

In der ersten Subgruppenanalyse wurde das Gesamtkollektiv in Patienten ohne operative Prozedur am Kolon ( $n=39)$, Patienten mit Kolonteilresektion $(n=8)$ und Patienten mit Kolektomie $(n=9)$ unterteilt. Die statistische Analyse der demographischen Daten und Komorbiditäten zeigte keine signifikanten Unterschiede zwischen den Subgruppen (Tabelle 10).

\begin{tabular}{|c|c|c|c|c|c|c|c|}
\hline & & \multirow[b]{2}{*}{$\begin{array}{c}\text { Kein } \\
\text { Koloneingriff }\end{array}$} & \multirow[b]{2}{*}{ Kolonteilresektion } & \multirow[b]{2}{*}{ Kolektomie } & \multicolumn{3}{|c|}{$p$} \\
\hline & & & & & $\begin{array}{l}\text { Kein Koloneingriff } \\
\text { vs. } \\
\text { Kolonteilresektion }\end{array}$ & $\begin{array}{c}\text { Kein } \\
\text { Koloneingriff } \\
\text { vs. } \\
\text { Kolektomie }\end{array}$ & $\begin{array}{c}\text { Kolonteilresektion } \\
\text { vs. } \\
\text { Kolektomie }\end{array}$ \\
\hline \multicolumn{2}{|l|}{$\mathrm{n}$} & 39 & 8 & 9 & & & \\
\hline \multicolumn{2}{|l|}{ Alter [Jahre] } & $56,9 \pm 14,6$ & $60,4 \pm 16,5$ & $55,9 \pm 16,6$ & 1,000 & 1,000 & 1,000 \\
\hline \multirow{2}{*}{ männlich } & $n$ & 23 & 4 & 5 & & \multirow{4}{*}{0,892} & \\
\hline & $\%$ & 59,0 & 50,0 & 55,6 & & & \\
\hline \multirow{2}{*}{ weiblich } & $n$ & 16 & 4 & 4 & & & \\
\hline & $\%$ & 41,0 & 50,0 & 44,4 & & & \\
\hline \multirow{2}{*}{ Hyperglykämie } & $n$ & 2 & 1 & 0 & & \multirow{2}{*}{0,531} & \\
\hline & $\%$ & 10,0 & 16,7 & 0,0 & & & \\
\hline \multirow{2}{*}{ Diabetes } & $\mathrm{n}$ & 7 & 3 & 1 & & \multirow{2}{*}{0,308} & \\
\hline & $\%$ & 35,0 & 50,0 & 12,5 & & & \\
\hline \multirow{2}{*}{ Hypertonie } & $n$ & 12 & 5 & 3 & & \multirow{2}{*}{0,223} & \\
\hline & $\%$ & 60,0 & 83,3 & 37,5 & & & \\
\hline \multirow{2}{*}{ Nikotinabusus } & $n$ & 6 & 1 & 1 & & \multirow{2}{*}{0,559} & \\
\hline & $\%$ & 30,0 & 16,7 & 12,5 & & & \\
\hline \multirow{2}{*}{ Alkoholabusus } & $\mathrm{n}$ & 5 & 0 & 2 & & \multirow{2}{*}{0,403} & \\
\hline & $\%$ & 23,8 & 0,0 & 25,0 & & & \\
\hline \multirow{2}{*}{ Adipositas } & $n$ & 7 & 2 & 2 & & \multirow{2}{*}{0,876} & \\
\hline & $\%$ & 35,0 & 33,3 & 25,0 & & & \\
\hline \multirow{2}{*}{ Hyperlipidämie } & $n$ & 3 & 2 & 0 & & \multirow{2}{*}{0,219} & \\
\hline & $\%$ & 15,0 & 33,3 & 0,0 & & & \\
\hline
\end{tabular}

Tabelle 10: Demographische Daten und Komorbiditäten. Daten werden als Mittelwerte \pm SD, absolute und relative Häufigkeiten dargestellt. 
Ferner zeigte die statistische Auswertung der Ätiologie der Pankreatitis keine Unterschiede zwischen den Subgruppen (Tabelle 11).

\begin{tabular}{|c|c|c|c|c|c|}
\hline & & Kein Koloneingriff & Kolonteilresektion & Kolektomie & $p$ \\
\hline \multirow{2}{*}{ Cholezystolithiasis } & $\mathrm{n}$ & 7 & 5 & 4 & \multirow{2}{*}{0,113} \\
\hline & $\%$ & 35,0 & 83,3 & 50,0 & \\
\hline \multirow{2}{*}{ Alkoholabusus } & $\mathrm{n}$ & 5 & 0 & 2 & \multirow{2}{*}{0,403} \\
\hline & $\%$ & 23,8 & 0,0 & 25,0 & \\
\hline \multirow{2}{*}{ post- ERCP } & $\mathrm{n}$ & 6 & 2 & 1 & \multirow{2}{*}{0,584} \\
\hline & $\%$ & 30,0 & 33,3 & 12,5 & \\
\hline \multirow{2}{*}{ Hyperlipidämie } & $\mathrm{n}$ & 3 & 2 & 0 & \multirow{2}{*}{0,219} \\
\hline & $\%$ & 15,0 & 33,3 & 0,0 & \\
\hline \multirow{2}{*}{ Hyperglykämie } & $\mathrm{n}$ & 2 & 1 & 0 & \multirow{2}{*}{0,531} \\
\hline & $\%$ & 10,0 & 16,7 & 0,0 & \\
\hline \multirow{2}{*}{ idiopathisch } & $\mathrm{n}$ & 3 & 0 & 0 & \multirow{2}{*}{0,316} \\
\hline & $\%$ & 15,0 & 0,0 & 0,0 & \\
\hline \multirow{2}{*}{ Rezidiv-Pankreatitis } & $\mathrm{n}$ & 4 & 2 & 1 & \multirow{2}{*}{0,512} \\
\hline & $\%$ & 10,3 & 25,0 & 11,1 & \\
\hline
\end{tabular}

Tabelle 11: Ätiologie. Daten werden als absolute und relative Häufigkeiten dargestellt.

Die Auswertung von sekundären Organkomplikationen und der Notwendigkeit von Organersatzverfahren wies ebenfalls keinerlei statistisch signifikante Differenzen zwischen den Teilkollektiven auf (Tabelle 12).

\begin{tabular}{|c|c|c|c|c|c|}
\hline & & Kein Koloneingriff & Kolonteilresektion & Kolektomie & $p$ \\
\hline \multirow{2}{*}{ Pneumonie/ARDS } & $\mathrm{n}$ & 9 & 3 & 2 & \multirow{2}{*}{0,555} \\
\hline & $\%$ & 45,0 & 50,0 & 25,0 & \\
\hline \multirow{2}{*}{ Beatmung } & $\mathrm{n}$ & 18 & 6 & 8 & \multirow{2}{*}{0,475} \\
\hline & $\%$ & 90,0 & 100,0 & 100,0 & \\
\hline \multirow{2}{*}{ akutes Nierenversagen } & $\mathrm{n}$ & 13 & 4 & 4 & \multirow{2}{*}{0,652} \\
\hline & $\%$ & 68,4 & 66,7 & 50,0 & \\
\hline \multirow{2}{*}{ Dialyse } & $\mathrm{n}$ & 11 & 3 & 5 & \multirow{2}{*}{0,890} \\
\hline & $\%$ & 55,0 & 50,0 & 62,5 & \\
\hline \multirow{2}{*}{ Leberversagen } & $\mathrm{n}$ & 5 & 1 & 1 & \multirow{2}{*}{0,735} \\
\hline & $\%$ & 25,0 & 16,7 & 12,5 & \\
\hline \multirow{2}{*}{$\begin{array}{l}\text { disseminierte intravasale } \\
\text { Koagulopathie }\end{array}$} & $\mathrm{n}$ & 3 & 1 & 0 & \multirow{2}{*}{0,495} \\
\hline & $\%$ & 15,0 & 16,7 & 0,0 & \\
\hline \multirow{2}{*}{ Transfusionsbedarf } & $\mathrm{n}$ & 19 & 6 & 7 & \multirow{2}{*}{0,596} \\
\hline & $\%$ & 95,0 & 100,0 & 87,5 & \\
\hline
\end{tabular}

Tabelle 12: Komplikationen und Organersatztherapie. Daten werden als absolute und relative Häufigkeiten dargestellt. 
Die statistische Auswertung der primären Endpunkte demonstrierte, dass Patienten nach Kolektomie im Vergleich zu Patienten ohne Koloneingriff eine deutlich geringere, wenngleich statistisch nicht signifikant geringere Krankenhaussterblichkeit aufwiesen (Tabelle 13). Für die 30- und 90-TagesMortalität zeigten sich ebenfalls keine signifikanten Unterschiede im Vergleich der Subgruppen.

\begin{tabular}{|c|c|c|c|c|c|}
\hline & & $\begin{array}{l}\text { Kein Koloneingriff } \\
\qquad(\mathrm{n}=39)\end{array}$ & $\begin{array}{l}\text { Kolonteilresektion } \\
\qquad(n=8)\end{array}$ & $\begin{array}{l}\text { Kolektomie } \\
\qquad(n=9)\end{array}$ & p \\
\hline \multirow{2}{*}{ Krankenhausmortalität } & $\mathrm{n}$ & 22 & 3 & 3 & \multirow{2}{*}{0,343} \\
\hline & $\%$ & 56,4 & 37,5 & 33,3 & \\
\hline \multirow{2}{*}{ 30-Tages-Mortalität } & $\mathrm{n}$ & 9 & 1 & 2 & \multirow{2}{*}{0,800} \\
\hline & $\%$ & 23,1 & 12,5 & 22,2 & \\
\hline \multirow{2}{*}{ 90-Tages-Mortalität } & $\mathrm{n}$ & 16 & 1 & 3 & \multirow{2}{*}{0,304} \\
\hline & $\%$ & 41,0 & 12,5 & 33,3 & \\
\hline
\end{tabular}

Tabelle 13: Primäre Endpunkte. Daten werden als absolute und relative Häufigkeiten dargestellt.

Die Analyse der sekundären Endpunkte (Tabelle 14) zeigte, dass die Krankenhausverweildauer bei Patienten mit einer Kolektomie oder Kolonteilresektion weitaus höher lag als bei Patienten ohne Koloneingriff. Unter denjenigen Patienten, die die akute nekrotisierende Pankreatitis überlebten, wiesen kolektomierte Patienten eine etwa doppelt so hohe Krankenhausverweildauer auf wie Patienten ohne Koloneingriff oder mit Kolonteilresektion $(p<0,05)$. Unter denjenigen Patienten, die innerhalb ihres Krankenhausaufenthaltes verstarben, zeigten sich signifikante Unterschiede. Kolektomierte Patienten verstarben durchschnittlich nach etwa 4 Wochen, Patienten ohne Koloneingriff überlebten doppelt so lange und Patienten mit Kolonteilresektion wiesen mit ungefähr 24 Wochen die weitaus längste Überlebenszeit im Krankenhaus auf.

\begin{tabular}{|c|c|c|c|c|c|c|c|}
\hline & \multirow[b]{2}{*}{$\begin{array}{l}\text { Kein } \\
\text { Kolon- } \\
\text { eingriff }\end{array}$} & \multirow[b]{2}{*}{$\begin{array}{l}\text { Kolonteil- } \\
\text { resektion }\end{array}$} & \multirow[b]{2}{*}{ Kolektomie } & \multirow[b]{2}{*}{$\mathbf{p}^{*}$} & \multicolumn{3}{|c|}{$p^{* *}$} \\
\hline & & & & & $\begin{array}{c}\text { Kein } \\
\text { Koloneingriff } \\
\text { vs. } \\
\text { Kolonteilresektion }\end{array}$ & $\begin{array}{c}\text { Kein } \\
\text { Koloneingriff } \\
\text { vs. } \\
\text { Kolektomie }\end{array}$ & $\begin{array}{c}\text { Kolonteil- } \\
\text { resektion } \\
\text { vs. } \\
\text { Kolektomie }\end{array}$ \\
\hline $\begin{array}{l}\text { Krankenhausverweildauer } \\
\text { insgesamt [d] }\end{array}$ & $61,1 \pm 43,1$ & $96,9 \pm 101,3$ & $86,6 \pm 54,3$ & 0,179 & 0,323 & 0,682 & 1,000 \\
\hline $\begin{array}{l}\text { Krankenhausverweildauer } \\
\text { der Überlebenden [d] }\end{array}$ & $64,4 \pm 37,1$ & $54,6 \pm 26,6$ & $116,3 \pm 37,2$ & 0,010 & 1,000 & 0,017 & 0,026 \\
\hline $\begin{array}{l}\text { Krankenhausverweildauer } \\
\text { der Verstorbenen [d] }\end{array}$ & $58,7 \pm 47,7$ & $167,3 \pm 150,4$ & $27,0 \pm 18,1$ & 0,016 & 0,024 & 1,000 & 0,029 \\
\hline Intensivverweildauer [d] & $40,5 \pm 40,3$ & $70,1 \pm 78,8$ & $52,9 \pm 23,7$ & 0,234 & 0,302 & 1,000 & 1,000 \\
\hline $\begin{array}{l}\text { Krankenhausverweildauer } \\
\text { vor } 1.0 P \text { [d] }\end{array}$ & $13,0 \pm 20,2$ & $6,9 \pm 10,7$ & $13,3 \pm 17,5$ & 0,691 & 1,000 & 1,000 & 1,000 \\
\hline $\begin{array}{l}\text { Intensivverweildauer vor } \\
1.0 P[d]\end{array}$ & $6,1 \pm 12,6$ & $3,0 \pm 6,1$ & $4,9 \pm 7,4$ & 0,775 & 1,000 & 1,000 & 1,000 \\
\hline
\end{tabular}

Tabelle 14: Sekundäre Endpunkte. Daten werden als Mittelwerte \pm SD dargestellt. * : Haupteffekt; **: post-hoc nach Bonferroni 


\subsubsection{Einfluss eines Koloneingriffs}

In dieser Subgruppenanalyse wurden die Patienten ohne jeglichen Eingriff am Kolon ( $n=39)$ mit denen verglichen, die einen Eingriff am Kolon erhalten hatten $(n=17)$, wobei für diesen Vergleich Patienten mit Kolonteilresektionen und Kolektomien zusammengefasst wurden.

\begin{tabular}{|c|c|c|c|c|}
\hline & & $\begin{array}{l}\text { Kein Koloneingriff } \\
\qquad(\mathrm{n}=39)\end{array}$ & $\begin{array}{l}\text { Koloneingriff } \\
\qquad(n=17)\end{array}$ & p \\
\hline \multirow{2}{*}{ Krankenhausmortalität } & $\mathrm{n}$ & 22 & 6 & \multirow{2}{*}{0,245} \\
\hline & $\%$ & 56,4 & 35,3 & \\
\hline \multirow{2}{*}{ 30-Tages-Mortalität } & $\mathrm{n}$ & 9 & 3 & \multirow{2}{*}{0,738} \\
\hline & $\%$ & 23,1 & 17,6 & \\
\hline \multirow{2}{*}{ 90-Tages-Mortalität } & $\mathrm{n}$ & 16 & 4 & \multirow{2}{*}{0,242} \\
\hline & $\%$ & 41,0 & 23,5 & \\
\hline
\end{tabular}

Tabelle 15: Primäre Endpunkte. Daten werden als absolute und relative Häufigkeiten dargestellt.

Die statistische Auswertung zeigte, dass die Patienten, die sich einem Koloneingriff unterziehen mussten, eine geringere Krankenhaussterblichkeit (35,3\%) aufwiesen als Patienten aus der Vergleichsgruppe (56,4\%), die keinen Eingriff am Kolon erhalten hatten. Dieser Unterschied war jedoch statistisch nicht signifikant. Ebenso wiesen 30- und 90-Tages-Mortalität keine signifikanten Unterschiede im Vergleich der beiden Subgruppen auf.

\begin{tabular}{|l|c|c|c|}
\hline & Kein Koloneingriff & Koloneingriff & $p$ \\
\hline Krankenhausverweildauer insgesamt [d] & $61,1 \pm 43,1$ & $91,4 \pm 77,4$ & 0,146 \\
\hline Krankenhausverweildauer der Überlebenden [d] & $64,4 \pm 37,1$ & $88,3 \pm 44,9$ & 0,145 \\
\hline Krankenhausverweildauer der Verstorbenen [d] & $58,7 \pm 47,7$ & $97,2 \pm 122,8$ & 0,483 \\
\hline Intensivverweildauer [d] & $40,5 \pm 40,3$ & $61,0 \pm 55,5$ & 0,128 \\
\hline Krankenhausverweildauer vor 1.OP [d] & $13,0 \pm 20,2$ & $10,3 \pm 14,6$ & 0,622 \\
\hline Intensivverweildauer vor 1.OP [d] & $6,1 \pm 12,6$ & $4,0 \pm 6,7$ & 0,530 \\
\hline
\end{tabular}

Tabelle 16: Sekundäre Endpunkte. Daten werden als Mittelwerte \pm SD dargestellt.

Patienten ohne jeglichen Eingriff am Kolon zeigten eine deutlich kürzere Krankenhaus- und Intensivverweildauer als die Patienten mit Koloneingriff. Unter denjenigen Patienten, die den Krankenhausaufenthalt bei akuter Pankreatitis überlebten, wiesen ebenfalls die Patienten ohne Koloneingriff eine wesentlich kürzere Krankenhausverweildauer auf als Patienten mit Kolektomie oder Kolonteilresektion. Patienten, die während des Krankenhausaufenthaltes verstarben und einen Koloneingriff erhalten hatte, lebten jedoch länger. Statistisch signifikante Unterschiede konnte diese Auswertung allerdings nicht demonstrieren. 


\subsubsection{Radikales Vorgehen: Einfluss der Kolektomie}

In dieser Subgruppenanalyse wurden diejenigen Patienten, die sich einer Kolektomie unterziehen mussten mit denjenigen Patienten verglichen, die keine Kolektomie erhalten hatten, eingeschlossen waren hier die Patienten mit Kolonteilresektion.

\begin{tabular}{|c|c|c|c|c|}
\hline & & $\begin{array}{l}\text { Keine Kolektomie } \\
\qquad(n=47)\end{array}$ & $\begin{array}{l}\text { Kolektomie } \\
\qquad(n=9)\end{array}$ & p \\
\hline \multirow{2}{*}{ Krankenhausmortalität } & $\mathrm{n}$ & 25 & 3 & \multirow{2}{*}{0,469} \\
\hline & $\%$ & 53,2 & 33,3 & \\
\hline \multirow{2}{*}{ 30-Tages-Mortalität } & $\mathrm{n}$ & 10 & 2 & \multirow{2}{*}{1,000} \\
\hline & $\%$ & 21,3 & 22,2 & \\
\hline \multirow{2}{*}{ 90-Tages-Mortalität } & $\mathrm{n}$ & 17 & 3 & \multirow{2}{*}{1,000} \\
\hline & $\%$ & 36,2 & 33,3 & \\
\hline
\end{tabular}

Tabelle 17: Primäre Endpunkte. Daten werden als absolute und relative Häufigkeiten dargestellt.

Die statistische Analyse der primären Endpunkte zeigte, dass Patienten nach Kolektomie im Vergleich zu nicht kolektomierten Patienten eine statistisch nicht signifikant geringere Mortalitätsrate aufwiesen. Dem gegenüber zeigte die statistische Auswertung der sekundären Endpunkte, dass kolektomierte Patienten eine deutlich längere Krankenhausverweildauer aufwiesen, die im Falle der überlebenden Patienten doppelt so lange war wie die der nicht-kolektomierten Patienten. Die Patienten, die an ihrer akuten nekrotisierenden Pankreatitis verstarben, verstarben trotz Kolektomie deutlich früher als die Patienten ohne Kolektomie (Tabelle 18).

\begin{tabular}{|l|c|c|c|}
\hline & Keine Kolektomie & Kolektomie & $p$ \\
\hline Krankenhausverweildauer insgesamt [d] & $67,3 \pm 57,6$ & $86,6 \pm 54,3$ & 0,359 \\
\hline Krankenhausverweildauer der Überlebenden [d] & $62,1 \pm 34,6$ & $116,3 \pm 37,2$ & 0,003 \\
\hline Krankenhausverweildauer der Verstorbenen [d] & $71,7 \pm 71,9$ & $27,0 \pm 18,1$ & 0,301 \\
\hline Intensivverweildauer [d] & $45,6 \pm 49,3$ & $52,9 \pm 23,7$ & 0,669 \\
\hline Krankenhausverweildauer vor 1.OP [d] & $11,9 \pm 19,0$ & $13,3 \pm 17,5$ & 0,839 \\
\hline Intensivverweildauer vor 1.OP [d] & $5,5 \pm 11,6$ & $4,9 \pm 7,4$ & 0,877 \\
\hline
\end{tabular}

Tabelle 18: Sekundäre Endpunkte. Daten werden als Mittelwerte \pm SD dargestellt. 


\subsubsection{Auswirkung der Durchführung einer Kolektomie im Rahmen des Ersteingriffs}

In diese Subgruppenanalyse wurden nur die Patienten mit Kolektomie eingeschlossen. Im Speziellen wurde untersucht, welche Auswirkung die Durchführung der Kolektomie im Rahmen des Ersteingriffs hat. Die Gesamtanzahl der kolektomierten Patienten betrug $n=9$, von ihnen wurden 5 im Rahmen des Ersteingriffs kolektomiert, 4 während eines späteren Eingriffs.

\begin{tabular}{|c|c|c|c|c|}
\hline & & $\begin{array}{l}\text { Kolektomie im Verlauf } \\
\qquad(n=4)\end{array}$ & $\begin{array}{l}\text { Kolektomie beim Ersteingriff } \\
\qquad(\mathrm{n}=5)\end{array}$ & p \\
\hline \multirow{2}{*}{ Krankenhausmortalität } & $\mathrm{n}$ & 2 & 1 & \multirow{2}{*}{0,524} \\
\hline & $\%$ & 50,0 & 20,0 & \\
\hline \multirow{2}{*}{ 30-Tages-Mortalität } & $\mathrm{n}$ & 2 & 0 & \multirow{2}{*}{0,167} \\
\hline & $\%$ & 50,0 & 0,0 & \\
\hline \multirow{2}{*}{ 90-Tages-Mortalität } & $n$ & 2 & 1 & \multirow{2}{*}{0,524} \\
\hline & $\%$ & 50,0 & 20,0 & \\
\hline
\end{tabular}

Tabelle 19: Primäre Endpunkte. Daten werden als absolute und relative Häufigkeiten dargestellt.

Die statistische Auswertung dieser Subgruppenanalyse demonstrierte, dass die Patienten, bei denen die Kolektomie im Rahmen des Ersteingriffs durchgeführt worden war, eine deutlich geringere Krankenhausmortalität sowie 30- und 90-Tages-Mortalität aufwiesen. Allerdings war der Unterschied zu den Patienten, die im Verlauf kolektomiert wurden, aufgrund der geringen Fallzahlen statistisch nicht signifikant.

\begin{tabular}{|l|c|c|c|}
\hline & Kolektomie im Verlauf & Kolektomie beim Ersteingriff & $p$ \\
\hline Krankenhausverweildauer insgesamt [d] & $81,8 \pm 74,6$ & $90,4 \pm 40,9$ & 0,844 \\
\hline Krankenhausverweildauer der Überlebenden [d] & $146,0 \pm 7,1$ & $101,5 \pm 37,6$ & 0,096 \\
\hline Krankenhausverweildauer der Verstorbenen [d] & $17,5 \pm 10,6$ & $46,0 \pm 0,0$ & 0,272 \\
\hline Intensivverweildauer [d] & $42,0 \pm 30,8$ & $61,6 \pm 14,2$ & 0,304 \\
\hline Krankenhausverweildauer vor 1.OP [d] & $5,0 \pm 7,3$ & $20,0 \pm 21,1$ & 0,222 \\
\hline Intensivverweildauer vor 1.OP [d] & $1,8 \pm 3,5$ & $7,4 \pm 9,0$ & 0,281 \\
\hline
\end{tabular}

Tabelle 20: Sekundäre Endpunkte. Daten werden als Mittelwerte \pm SD dargestellt.

Die Auswertung der sekundären Endpunkte zeigte, dass die überlebenden Patienten eine deutlich niedrigere Krankenhausverweildauer aufweisen, wenn sie die Kolektomie bei Ersteingriff erhalten hatten. Diejenigen Patienten, die an der akuten nekrotisierenden Pankreatitis verstarben, verstarben deutlich später, sofern die Kolektomie beim Ersteingriff durchgeführt worden war (46,0 Tage vs. 17,5 Tage). Aufgrund der geringen Fallzahlen konnten auch hier keine statistisch signifikanten Unterschiede aufgezeigt werden. 


\subsubsection{Stellenwert einer frühzeitigen Kolektomie}

Im Rahmen dieser Subgruppenanalyse wurden ebenfalls nur die Patienten analysiert, die sich einer Kolektomie unterziehen mussten. Hier wurde die Frage untersucht, ob das Zeitintervall zwischen der Krankenhausaufnahme des Patienten und der Durchführung der Kolektomie für das Ergebnis der Patienten von Bedeutung ist. Als Grenzwert zwischen frühzeitiger und später Kolektomie wurde eine Zeitdauer zwischen Aufnahmedatum und Kolektomie von 7 Tagen gewählt.

\begin{tabular}{|c|c|c|c|c|}
\hline & & $\begin{array}{c}\text { Kolektomie } \leq 7 \text { Tage } \\
\qquad(n=3)\end{array}$ & $\begin{array}{l}\text { Kolektomie }>7 \text { Tage } \\
\qquad(n=6)\end{array}$ & $\mathbf{p}$ \\
\hline \multirow{2}{*}{ Krankenhausmortalität } & $n$ & 1 & 2 & \multirow{2}{*}{1,000} \\
\hline & $\%$ & 33,3 & 33,3 & \\
\hline \multirow{2}{*}{ 30-Tages-Mortalität } & $\mathrm{n}$ & 1 & 1 & \multirow{2}{*}{1,000} \\
\hline & $\%$ & 33,3 & 16,7 & \\
\hline \multirow{2}{*}{ 90-Tages-Mortalität } & $n$ & 1 & 2 & \multirow{2}{*}{1,000} \\
\hline & $\%$ & 33,3 & 33,3 & \\
\hline
\end{tabular}

Tabelle 21: Primäre Endpunkte. Daten werden als absolute und relative Häufigkeiten dargestellt.

Die statistische Auswertung der Krankenhausmortalität zeigte keinen statistischen Unterschied zwischen Patienten, die frühzeitig oder nach mehr als 7 Tagen kolektomiert wurden. Auch der Vergleich der 30- bzw. 90-Tages-Mortalitäten der beiden Vergleichsgruppen zeigte keine Signifikanzen.

\begin{tabular}{|l|c|c|c|}
\hline & Kolektomie $\leq 7$ Tage & Kolektomie > 7 Tage & $p$ \\
\hline Krankenhausverweildauer insgesamt [d] & $74,0 \pm 65,6$ & $92,8 \pm 53,4$ & 0,655 \\
\hline Krankenhausverweildauer der Überlebenden [d] & $106,0 \pm 49,5$ & $121,5 \pm 37,2$ & 0,683 \\
\hline Krankenhausverweildauer der Verstorbenen [d] & $10,0 \pm 0,0$ & $35,5 \pm 14,8$ & 0,394 \\
\hline Intensivverweildauer [d] & $46,3 \pm 33,2$ & $56,2 \pm 20,5$ & 0,593 \\
\hline Krankenhausverweildauer vor 1.OP [d] & $1,0 \pm 1,0$ & $19,5 \pm 18,8$ & 0,143 \\
\hline Intensivverweildauer vor 1.OP [d] & $0,0 \pm 0,0$ & $7,3 \pm 8,1$ & 0,173 \\
\hline
\end{tabular}

Tabelle 22: Sekundäre Endpunkte. Daten werden als Mittelwerte \pm SD dargestellt.

Diejenigen Patienten, die nach mehr als 7 Tagen kolektomiert worden waren, hatten eine längere, wenngleich statistisch nicht signifikante Krankenhaus- und Intensivverweildauer.

Im Rahmen dieser Subgruppenanalyse wurde in einer zweiten Auswertung die Frage untersucht, ob das Zeitintervall zwischen der Diagnosestellung der akuten nekrotisierenden Pankreatitis und der Durchführung der Kolektomie für das Resultat der Patienten von Bedeutung ist. Als Grenzwert wurde wiederum eine Zeitdauer zwischen Diagnose und Kolektomie von 7 Tagen gewählt (Tabelle 23). 


\begin{tabular}{|c|c|c|c|c|}
\hline & & $\begin{array}{l}\text { Kolektomie } \leq 7 \text { Tage nach Diagnose } \\
\qquad(n=4)\end{array}$ & $\begin{array}{c}\text { Kolektomie }>7 \text { Tage nach } \\
\text { Diagnose } \\
(n=5)\end{array}$ & p \\
\hline \multirow{2}{*}{ Krankenhausmortalität } & $n$ & 1 & 2 & \multirow{2}{*}{1,000} \\
\hline & $\%$ & 25,0 & 40,0 & \\
\hline \multirow{2}{*}{ 30-Tages-Mortalität } & $n$ & 1 & 1 & \multirow{2}{*}{1,000} \\
\hline & $\%$ & 25,0 & 20,0 & \\
\hline \multirow{2}{*}{ 90-Tages-Mortalität } & $\mathrm{n}$ & 1 & 2 & \multirow{2}{*}{1,000} \\
\hline & $\%$ & 25,0 & 40,0 & \\
\hline
\end{tabular}

Tabelle 23: Primäre Endpunkte. Daten werden als absolute und relative Häufigkeiten dargestellt.

Die statistische Auswertung der primären Endpunkte zeigte keine statistisch signifikanten Unterschiede zwischen denjenigen Patienten, die die Kolektomie innerhalb von 7 Tagen nach Diagnosestellung und denjenigen Patienten, die Kolektomie zu einem späteren Zeitpunkt erhalten hatten.

\begin{tabular}{|l|c|c|} 
& Kolektomie $\leq 7$ Tage nach Diagnose & $\begin{array}{c}\text { Kolektomie }>7 \text { Tage nach } \\
\text { Diagnose }\end{array}$ \\
\hline Krankenhausverweildauer insgesamt [d] & $89,5 \pm 61,9$ & $84,2 \pm 54,8$ \\
\hline Krankenhausverweildauer der Überlebenden [d] & $116,0 \pm 39,1$ & $116,7 \pm 44,0$ \\
\hline Krankenhausverweildauer der Verstorbenen [d] & $10,0 \pm 0,0$ & $35,5 \pm 14,8$ \\
\hline Intensivverweildauer [d] & $55,8 \pm 33,0$ & 0,985 \\
\hline Krankenhausverweildauer vor 1.OP [d] & $3,0 \pm 4,1$ & 0,394 \\
\hline Intensivverweildauer vor 1.OP [d] & $1,5 \pm 3,0$ & $21,6 \pm 20,2$ \\
\hline
\end{tabular}

Tabelle 24: Sekundäre Endpunkte. Daten werden als Mittelwerte \pm SD dargestellt.

Auch in Bezug auf die Krankenhausverweildauer aller kolektomierten Patienten sowie der Überlebenden zeigten sich keine Unterschiede im Subgruppenvergleich. Allerdings zeigte diese Auswertung, dass die Patienten, die innerhalb der ersten sieben Tage nach Diagnosestellung kolektomiert wurden, deutlich früher verstarben als die Patienten, die später als sieben Tage nach Diagnosestellung kolektomiert wurden. Aufgrund der geringen Fallzahlen konnten jedoch auch hier keine signifikanten Unterschiede aufgezeigt werden. 


\subsubsection{Zentrumsübergreifende Analyse des Stellenwertes der Kolektomie}

Analysiert wurden hier drei Teilkollektive. Diese wurden gebildet aus dem gesamten Homburger Kollektiv, einer Subgruppe bestehend aus Homburger Patienten, die nicht kolektomiert worden waren und dem gesamten Göttinger Kollektiv.

\begin{tabular}{|c|c|c|c|c|c|}
\hline & & $\begin{array}{c}\text { HOM alle } \\
(n=37)\end{array}$ & $\begin{array}{l}\text { HOM ohne Kolektomie } \\
\qquad(\mathrm{n}=28)\end{array}$ & $\begin{array}{l}\text { GÖ alle } \\
(n=19)\end{array}$ & p \\
\hline \multirow{2}{*}{ Krankenhausmortalität } & $\mathrm{n}$ & 18 & 15 & 10 & \multirow{2}{*}{0,916} \\
\hline & $\%$ & 48,6 & 53,6 & 52,6 & \\
\hline \multirow{2}{*}{ 30-Tages-Mortalität } & $\mathrm{n}$ & 7 & 5 & 5 & \multirow{2}{*}{0,751} \\
\hline & $\%$ & 18,9 & 17,9 & 26,3 & \\
\hline \multirow{2}{*}{ 90-Tages-Mortalität } & $\mathrm{n}$ & 13 & 10 & 7 & \multirow{2}{*}{0,992} \\
\hline & $\%$ & 35,1 & 35,7 & 36,8 & \\
\hline
\end{tabular}

Tabelle 25: Primäre Endpunkte. Daten werden als absolute und relative Häufigkeiten dargestellt.

Die Krankenhausmortalität sowie 30- und 90-Tages-Mortalitäten waren in allen drei Subgruppen vergleichbar und ohne statistisch signifikante Unterschiede.

\begin{tabular}{|l|l|l|l|l|l|l|c|}
\hline & HOM alle & HOM ohne Kolektomie & GÖ alle & $p^{*}$ & $\begin{array}{c}\text { HOM alle } \\
\text { vs. } \\
\text { HOM ohne } \\
\text { Kolektomie }\end{array}$ & $\begin{array}{c}\text { HOM } \\
\text { alle } \\
\text { vs. } \\
\text { G0̈ alle }\end{array}$ & $\begin{array}{c}\text { HOM ohne } \\
\text { Kolektomie } \\
\text { vs. } \\
\text { G0̈ alle }\end{array}$ \\
\hline $\begin{array}{l}\text { Krankenhausverweildauer insgesamt } \\
\text { [d] }\end{array}$ & $74,7 \pm 60,7$ & $70,7 \pm 63,2$ & $62,5 \pm 49,7$ & 0,768 & 1,000 & 1,000 & 1,000 \\
\hline $\begin{array}{l}\text { Krankenhausverweildauer der } \\
\text { Überlebenden [d] }\end{array}$ & $75,9 \pm 41,3$ & $55,7 \pm 25,9$ & $70,7 \pm 43,8$ & 0,363 & 0,438 & 1,000 & 1,000 \\
\hline $\begin{array}{l}\text { Krankenhausverweildauer der } \\
\text { Verstorbenen [d] }\end{array}$ & $73,5 \pm 76,7$ & $82,8 \pm 80,9$ & $55,1 \pm 55,8$ & 0,658 & 1,000 & 1,000 & 1,000 \\
\hline Intensivverweildauer [d] & $49,7 \pm 47,9$ & $48,6 \pm 53,9$ & $41,5 \pm 43,0$ & 0,832 & 1,000 & 1,000 & 1,000 \\
\hline Krankenhausverweildauer vor 1.OP [d] & $13,4 \pm 21,1$ & $13,5 \pm 22,5$ & $9,7 \pm 12,7$ & 0,777 & 1,000 & 1,000 & 1,000 \\
\hline Intensivverweildauer vor 1.OP [d] & $6,1 \pm 12,2$ & $6,6 \pm 13,6$ & $4,1 \pm 8,6$ & 0,764 & 1,000 & 1,000 & 1,000 \\
\hline
\end{tabular}

Tabelle 26: Sekundäre Endpunkte. Daten werden als Mittelwerte \pm SD dargestellt. * : Haupteffekt; **: post-hoc nach Bonferroni

Die Auswertung der sekundären Endpunkte zeigte, dass die Krankenhausverweildauer im Homburger Gesamtkollektiv mit 75 Tagen am höchsten lag. Etwas niedriger war sie bei den Homburger Patienten, die keine Kolektomie erhalten hatten, die kürzeste Krankenhausverweildauer zeigten die Göttinger Patienten. Beim Vergleich der Krankenhausverweildauer der Überlebenden fiel auf, dass die Homburger Patienten ohne Kolektomie mit 55,7 Tagen einen deutlich kürzeren Krankenhausaufenthalt hatten. Gleichzeitig hatten diejenigen Patienten, die an der akuten nekrotisierenden Pankreatitis 
verstarben, in diesem Kollektiv die längste Überlebensdauer im Krankenhaus. Letztlich zeigte die statistische Auswertung keine signifikanten Unterschiede zwischen den gewählten Subgruppen.

In einer weiteren Auswertung wurden ebenfalls drei Teilkollektive gebildet. Ziel war ein Vergleich zwischen dem Homburger Kollektiv ohne Kolektomie ( $n=28)$, dem Homburger Kollektiv mit Kolektomie $(n=9)$ und dem gesamten Göttinger Kollektiv $(n=19)$.

\begin{tabular}{|c|c|c|c|c|c|c|}
\hline & & $\begin{array}{l}\text { HOM ohne Kolektomie } \\
\qquad(\mathrm{n}=28)\end{array}$ & $\begin{array}{l}\text { HOM mit Kolektomie } \\
\qquad(\mathrm{n}=9)\end{array}$ & $\begin{array}{l}\text { GÖ alle } \\
(n=19)\end{array}$ & $\mathbf{p}$ & $\begin{array}{c}\text { p } \\
\text { (HOM ohne Kolektomie } \\
\text { vs. GÖ alle) }\end{array}$ \\
\hline \multirow{2}{*}{ Krankenhausmortalität } & $\mathrm{n}$ & 15 & 3 & 10 & \multirow{2}{*}{0,550} & \multirow{2}{*}{1,000} \\
\hline & $\%$ & 53,6 & 33,3 & 52,6 & & \\
\hline \multirow{2}{*}{ 30-Tages-Mortalität } & $\mathrm{n}$ & 5 & 2 & 5 & \multirow{2}{*}{0,785} & \multirow{2}{*}{0,496} \\
\hline & $\%$ & 17,9 & 22,2 & 26,3 & & \\
\hline \multirow{2}{*}{ 90-Tages-Mortalität } & $\mathrm{n}$ & 10 & 3 & 7 & \multirow{2}{*}{0,984} & \multirow{2}{*}{1,000} \\
\hline & $\%$ & 35,7 & 33,3 & 36,8 & & \\
\hline
\end{tabular}

Tabelle 27: Primäre Endpunkte. Daten werden als absolute und relative Häufigkeiten dargestellt.

Die höchste Krankenhaussterblichkeit wies mit 53,6 \% das Teilkollektiv der Homburger Patienten ohne Kolektomie auf, im Göttinger Gesamtkollektiv lag sie bei 52,6 \%. In der Gruppe der kolektomierten Homburger Patienten verstarben nur 33,3\% der Patienten, wenngleich der Unterschied nicht signifikant war $(p>0,05)$. Auch der Vergleich der 30- und 90-Tages-Mortalitäten über die drei Subgruppen zeigte keine statistisch signifikanten Unterschiede. Ebenso ergab der isolierte Vergleich der Homburger Patienten ohne Kolektomie mit dem Göttinger Gesamtkollektiv keine signifikanten Differenzen.

\begin{tabular}{|c|c|c|c|c|c|c|c|c|}
\hline & \multirow[b]{2}{*}{$\begin{array}{l}\text { HOM ohne } \\
\text { Kolektomie }\end{array}$} & \multirow[b]{2}{*}{$\begin{array}{l}\text { HOM mit } \\
\text { Kolektomie }\end{array}$} & \multirow[b]{2}{*}{ GÖ alle } & \multirow[b]{2}{*}{$\mathrm{p}^{*}$} & \multicolumn{3}{|c|}{$p^{* *}$} & \multirow[b]{2}{*}{$\begin{array}{c}\text { p } \\
\text { (HOM ohne } \\
\text { Kolektomie } \\
\text { vs. } \\
\text { GÖ alle) }\end{array}$} \\
\hline & & & & & $\begin{array}{l}\text { HOM ohne } \\
\text { vs. HOM } \\
\text { mit } \\
\text { Kolektomie }\end{array}$ & $\begin{array}{c}\text { HOM ohne } \\
\text { Kolektomie } \\
\text { vs. } \\
\text { GÖ alle }\end{array}$ & $\begin{array}{l}\text { HOM mit } \\
\text { Kolektomie } \\
\text { vs. } \\
\text { GÖ alle }\end{array}$ & \\
\hline $\begin{array}{l}\text { Krankenhausverweildauer } \\
\text { insgesamt [d] }\end{array}$ & $70,7 \pm 63,2$ & $86,6 \pm 54,3$ & $62,5 \pm 49,7$ & 0,588 & 1,000 & 1,000 & 0,619 & 0,623 \\
\hline $\begin{array}{l}\text { Krankenhausverweildauer } \\
\text { der Überlebenden [d] }\end{array}$ & $55,7 \pm 25,9$ & $116,3 \pm 37,2$ & $70,7 \pm 43,8$ & 0,008 & 0,006 & 1,000 & 0,064 & 0,338 \\
\hline $\begin{array}{l}\text { Krankenhausverweildauer } \\
\text { der Verstorbenen [d] }\end{array}$ & $82,8 \pm 80,9$ & $27,0 \pm 18,1$ & $55,1 \pm 55,8$ & 0,370 & 0,645 & 1,000 & 1,000 & 0,356 \\
\hline Intensivverweildauer [d] & $48,6 \pm 53,9$ & $52,9 \pm 23,7$ & $41,5 \pm 43,0$ & 0,804 & 1,000 & 1,000 & 1,000 & 0,637 \\
\hline $\begin{array}{l}\text { Krankenhausverweildauer } \\
\text { vor } 1.0 \mathrm{P}[\mathrm{d}]\end{array}$ & $13,5 \pm 22,5$ & $13,3 \pm 17,5$ & $9,7 \pm 12,7$ & 0,787 & 1,000 & 1,000 & 1,000 & 0,515 \\
\hline $\begin{array}{l}\text { Intensivverweildauer vor } \\
1.0 P \text { [d] }\end{array}$ & $6,6 \pm 13,6$ & $4,9 \pm 7,4$ & $4,1 \pm 8,6$ & 0,750 & 1,000 & 1,000 & 1,000 & 0,482 \\
\hline
\end{tabular}

Tabelle 28: Sekundäre Endpunkte. Daten werden als Mittelwerte \pm SD dargestellt. * : Haupteffekt; **: post-hoc nach Bonferroni 
Das Teilkollektiv der kolektomierten Homburger Patienten zeigte die längste Krankenhausverweildauer. Dieser Unterschied wurde insbesondere bei den überlebenden Patienten evident. Die Auswertung der Krankenhausverweildauer der verstorbenen Patienten zeigte für diese Gruppe hingegen die kürzeste Überlebensdauer (27,0 Tage), obgleich der Unterschied statistisch nicht signifikant war (Tabelle 28). Auch der isolierte statistische Vergleich zwischen den Homburger Patienten ohne Kolektomie und den Göttinger Patienten konnte keine signifikanten Unterschiede zwischen den beiden Teilkollektiven demonstrieren. 


\section{Diskussion}

Kolonnekrosen und -perforationen gelten als schwerwiegende Komplikationen der akuten nekrotisierenden Pankreatitis. Mit einer Inzidenz von etwa $15 \%$ sind sie in der Regel mit einem fulminanten Verlauf der Erkrankung assoziiert (Van Minnen et al. 2004).

Während die Pathomechanismen, die zu einem Überschreiten des Entzündungsprozesses auf das Kolon führen, noch nicht abschließend geklärt sind, herrscht allgemeiner Konsens darüber, dass es bei gestörter Barrierefunktion der Darmmukosa zu einer Kontamination der Nekroseareale des Pankreas mit enterischen Bakterien kommt. Superinfektionen der Pankreasnekrosen mit daraus resultierender Sepsis, sekundäre Organdysfunktionen und - schäden bis hin zu Multiorganversagen können die Folge sein und sind mit einer hohen Mortalität verbunden (Nagpal et al. 2015).

Im Rahmen dieser retrospektiven statistischen Analyse sollte daher die Frage beantwortet werden, ob ein radikal chirurgisches Vorgehen inklusive der Entfernung des gesamten Kolons das Outcome der Patienten mit akuter nekrotisierender Pankreatitis verbessern kann.

Obwohl erste Erkenntnisse über Kolonkomplikationen bei akuter Pankreatitis bereits im Jahr 1967 gewonnen wurden (Lukash 1967), sind bisher nur wenige Studien zu dem Thema der zusätzlichen Resektion des Kolons bei akuter nekrotisierender Pankreatitis veröffentlicht worden.

Die vorliegende Studie basiert auf Patientendaten zweier deutscher universitärer chirurgischer Zentren, dem Universitätsklinikum des Saarlandes in Homburg/Saar und dem Universitätsklinikum Göttingen.

Während im Universitätsklinkum des Saarlandes das Konzept der Kolektomie in das chirurgische Management der akuten nekrotisierenden Pankreatitis im Beobachtungszeitraum fest implementiert war, wurde dieses Verfahren in der Universitätsmedizin Göttingen bei Patienten mit dieser Erkrankung nur gegebenenfalls als Teilresektion des Kolons durchgeführt.

Primäre Endpunkte, die für den Vergleich herangezogen wurden, waren die Krankenhausmortalität sowie die 30- und 90-Tages-Mortalität. Diese Parameter wurden als valide, standardisierte und etablierte Messgrößen des Therapieerfolges gewählt. Als weitere Surrogatparameter dienten die Dauer des Klinikaufenthaltes insgesamt, die Verweildauer auf der Intensivstation und der Zeitraum des Aufenthaltes vor der 1. Operation sowohl auf der Intensivstation als auch auf der Normalstation. Sämtliche Daten wurden aus den zur Verfügung stehenden digitalisierten und papierbasierten Patientenakten erhoben. 
Es wurden insgesamt 72 Patienten in die Studie einbezogen, 38 aus Homburg/Saar und 34 aus Göttingen. Aufgrund mangelnder Schwere der Erkrankung wurde nochmals eine Selektion durchgeführt, wobei Kreislaufinsuffizienz mit Katecholaminpflichtigkeit als Voraussetzung für den Einschluss in die Studie festgesetzt wurde. Schlussendlich ergab sich daraus eine Kohorte von 56 Patienten, die einen fulminanten Verlauf der akuten nekrotisierenden Pankreatitis zeigten. Diese wurden der statistischen Analyse zugeführt.

Gemäß den Leitlinien wird derzeit ein primär konservatives Management bei der Behandlung der akuten nekrotisierenden Pankreatitis empfohlen, erst bei Nachweis infizierter Nekrosen sollte chirurgisch interveniert werden. So zeigte eine Studie bei rein konservativer Behandlung von Patienten mit infizierten Pankreasnekrosen eine Sterblichkeitsrate von bis zu $100 \%$, während die chirurgische Behandlung von Patienten mit einer derartigen Schwere der Erkrankung diese deutlich auf 10 bis $30 \%$ senken konnte (Büchler et al. 2000, Widdison und Karanjia 1993). Die operative Therapie beschränkt sich meist auf das Entfernen superinfizierter Nekroseareale, da diese als Trigger für systemische Komplikationen verantwortlich gemacht werden (Besselink et al. 2013, Uhl et al. 2002). Die zusätzliche Resektion des Kolons hat sich dabei nicht durchgesetzt, obwohl das Kolon bereits seit Langem als Quelle der bakteriellen Kontamination der Nekroseareale angesehen wird (Kriwanek et al. 1994).

Da die radikale chirurgische Therapie einer zusätzlichen Kolektomie nur bei dem Krankheitsbild einer maximal schweren akuten nekrotisierenden Pankreatitis an Bedeutung gewinnt, wurden eine intensivmedizinische Behandlung, die Notwendigkeit eines chirurgischen Eingriffs sowie die Katecholaminpflichtigkeit bei Kreislaufinsuffizienz als Aufnahmekriterien der Patienten in diese Studie festgelegt. Anhand dieser sicherlich kleinen Gruppe von Patienten mit einer schlechten Prognose der Erkrankung sollte der Stellenwert einer ausgedehnten chirurgischen Therapie durch Nekrosektomie mit zusätzlicher Resektion des Kolons evaluiert werden.

Das Gesamtkollektiv bestand aus 56 Patienten, die sich aus 37 Homburger und 19 Göttinger Patienten zusammensetzen. Die Geschlechterverteilung entsprach den Erwartungen, Männer waren mit ungefähr $60 \%$ etwas häufiger betroffen als Frauen. Der Altersdurchschnitt lag mit 57 Jahren hingegen über dem in der Literatur beschriebenen Erkrankungsgipfel, wonach die meisten Menschen bereits weitaus früher zwischen dem 35. und 44. Lebensjahr an der akuten nekrotisierenden Pankreatitis erkranken (Lankisch et al. 2002). Begleiterkrankungen wie Hypertonie (58,5\%), Diabetes (32,4\%) und Adipositas (32,4\%) waren häufig und bestätigten die aktuelle Zunahme von Zivilisationskrankheiten im Wandel der gesellschaftlichen Entwicklung. Das erhöhte Risiko einer Hyperlipidämie bei Adipositas-Patienten und 
die Korrelation zwischen Hyperglykämie und einem Diabetes mellitus zeigte eine enge Verzahnung von Begleiterkrankungen und ätiologischen Faktoren der akuten nekrotisierenden Pankreatitis. Die Hyperlipidämie sowie die Hyperglykämie wurden in dieser Studie als feste Parameter sowohl bei Komorbiditäten als auch bei ätiologischen Faktoren dieser Erkrankung aufgeführt.

Die Ätiologie zeigte eine literaturkonforme Verteilung, wonach Gallensteinleiden und Alkoholabusus zu den häufigsten Ursachen einer akuten nekrotisierenden Pankreatitis zählen (Spanier et al. 2008). Der Anteil der post-ERCP-induzierten akuten nekrotisierenden Pankreatitis lag jedoch mit ungefähr $25 \%$ höher als in den Industrieländern verbreitet, und nahm in dieser Studie noch vor der äthyltoxisch induzierten akuten Pankreatitis Platz zwei der Ätiologie ein.

Weshalb die Häufigkeit einer post-interventionell indizierten akuten nekrotisierenden Pankreatitis von der derzeitig in der Literatur angegebenen Häufigkeit abweicht, kann nur aufgrund der im Rahmen dieser Analyse erhoben Daten nicht beantwortet werden. Eine Bewertung dieses Parameters stand ebenso nicht im Fokus dieser Analyse. Eventuell handelte es sich bei den Patienten mit postinterventioneller Pankreatitis um komplexere Krankheitsfälle, die ohnehin ein höheres Risiko von Komplikationen mit sich führten. Ebenso kann über die Expertise des Endoskopikers und dessen Ausbildungsstand und damit assoziierte Routine beziehungsweise dessen persönliche Komplikationshäufigkeit keine Aussage getroffen werden. Auch bleibt unklar, ob eine ERCP im vorliegenden Patientenkollektiv häufiger bzw. frühzeitiger durchgeführt wurde. Fest steht hingegen, dass die akute Pankreatitis mit 5,4 \% eine der häufigsten Komplikationen der endoskopisch retrograden Cholangiopankreatikographie darstellt (Amelsberg 1997). Andere ätiologische Faktoren wie die Hyperlipidämie als Stoffwechselerkrankung, Virusinfektionen oder abdominelle Traumata wurden seltener oder gar nicht diagnostiziert.

Die Analyse der sekundären Organkomplikationen bestätigte die maximale Schwere der akuten nekrotisierenden Pankreatitis im vorliegenden Patientenkollektiv. Pulmonale Komplikationen in Form einer Pneumonie konnten in etwa $40 \%$, ein akutes Nierenversagen bei annähernd $65 \%$ der Fälle beobachtetet werden. Organersatzverfahren in Form einer Beatmung waren bei fast $95 \%$ notwendig, dialysepflichtig wurden etwas mehr als die Hälfte der Patienten und eine Transfusion aufgrund einer Knochenmarksdysfunktion erhielten ebenfalls fast $95 \%$ der Patienten.

Diese Ergebnisse decken sich mit der Studienlage, wonach eine Pankreatitis-assoziierte Sepsis nicht selten mit Schockzuständen, intravasalem Volumenmangel sowie respiratorischer und renaler Insuffizienz assoziiert ist. Infarkttypische EKG-Veränderungen sind zwar häufig, eigentliche kardiale Komplikationen wie Perikardergüsse oder Herzbeuteltamonaden werden jedoch als äußerst selten in 
Verbindung mit der akuten nekrotisierenden Pankreatitis gebracht. Dies deckt sich wiederum mit dem Kollektiv dieser Studie, in dem neben der als Einschlusskriterium postulierten kardiozirkulatorischen Dysfunktion kein Patient mit sonstiger kardialer Sekundärkomplikation im Sinne eines Infarktes oder Perikardergusses beobachtet wurde. Systemische Komplikationen beeinflussen den Verlauf der akuten Pankreatitis entscheidend und gelten somit als signifikanter Faktor für die hohe Mortalität der akuten nekrotisierenden Pankreatitis (Beger et al. 1997, Besselink et al. 2009, Bradley 1993).

Nach Auswertung der unterschiedlichen chirurgischen Therapiemaßnahmen im Gesamtkollektiv erhielten 69,6 \% der Patienten eine alleinige Nekrosektomie ohne Eingriff am Kolon. Im Rahmen der chirurgischen Fokussanierung mussten sich 16,1 \% der Patienten einer Kolektomie und 14,3 \% einer Kolonteilresektion unterziehen. Bei vergleichbarem Patientengut wurde in Homburg bei $24,3 \%$ der Patienten eine Kolektomie durchgeführt. Die Recherche und nachfolgende Selektion konnte hingegen im Göttinger Kollektiv keinen kolektomierten Patienten mit einer schweren Form der akuten nekrotisierenden Pankreatitis herausfiltern, was bestätigt, dass das Konzept der zusätzlichen Kolektomie in der Therapie der akuten nekrotisierenden Pankreatitis in Göttingen nicht etabliert ist. Die Analyse zeigte ebenfalls, dass die Homburger Patienten im Durchschnitt häufiger operiert wurden als die Göttinger Patienten. Der Grund für die häufigeren Operationen der Patienten in Homburg kann letztlich anhand der vorliegenden Akten nicht genau rekonstruiert werden. Insbesondere der Grund für die deutlich höhere Zahl an Eingriffen in Homburg im Vergleich zu Göttingen sowie die Indikation für ein gegebenenfalls aggressives Management in Form einer Kolektomie lassen sich retrospektiv nicht analysieren. Die höhere Anzahl an Operationen könnte sowohl Ausdruck einer initial zurückhaltenden und schonenden chirurgischen Herangehensweise sein, es kann jedoch auch hypothetisiert werden, dass trotz der statistisch gegebenen Vergleichbarkeit der Kollektive die Homburger Patienten deutlich kränker waren als die Göttinger Patienten und deshalb öfter chirurgischer Maßnahmen bedurften.

Übereinstimmend mit der Studienlage ergab sich im vorliegenden Gesamtkollektiv eine Krankenhausmortalität von $50 \%$, im Homburger Grundkollektiv verstarben 48,6 \%, im Göttinger Grundkollektiv lag die Krankenhaussterblichkeit bei 52,6 \% (Bank et al. 2002).

Für diese minimalen Unterschiede der Teilkollektive könnten patienteneigene Faktoren wie beispielsweise chronische Vorerkrankungen, wie sie beim APACHE-Score zur Risikoeinschätzung erhoben werden, eine Rolle spielen. Dieser war der retrospektiven Analyse aufgrund nicht verfügbarer Parameter jedoch nicht zu ermitteln, sodass über etwaige Unterschiede bezüglich des APACHE-Scores keine Aussagen getroffen werden können.

Die Subgruppenauswertung, die den Einfluss der verschiedenen chirurgischen Therapiekonzepte zentrumsübergreifend analysieren sollte, zeigte, dass die Krankenhausmortalität der Patienten ohne 
Koloneingriff bei $56,4 \%$, bei Patienten mit Kolektomie nur bei 33,3 \% lag. Aufgrund der geringen Fallzahl ist jedoch eine aus diesem Ergebnis resultierende Beurteilung zunächst wenig aussagekräftig, der Unterschied erreichte zudem in der statistischen Analyse keine Signifikanz. Ferner ist über die Ausgangslage der Patienten und die Schwere der Erkrankung aufgrund des nicht vorliegenden APACHE-Scores keine Aussage möglich.

Dennoch stellt die aus dieser Analyse gewonnene Aussage einer deutlichen Reduktion der Krankenhausmortalität nach Kolektomie zunächst einen tendenziell für diese Therapieoption sprechenden Faktor dar.

Bei der allgemeinen Diskussion über Vorteile einer zusätzlichen Kolektomie dürfen jedoch Folgezustände eines aggressiven chirurgischen Vorgehens nicht außer Acht gelassen werden. Postoperative Komplikationen wie eine gestörte Wundheilung, Blutungen, Anastomosen-Insuffizienz nach Kontinuitätswiederherstellung, Enterostomie-assoziierte Probleme und die Ausbildung eines postoperativen lleus haben einen erheblichen Einfluss auf die Prognose der Patienten (Smith et al. 2004). Sie werden bei elektiv operierten Patienten ohne Pankreatitis-assoziierte, septische sekundäre Organdysfunktionen und Mikrozirkulationsstörungen mit einer Häufigkeit von bis zu 11 \% beschrieben und haben eine Steigerung von Morbidität und Mortalität zur Folge (Daams et al. 2013, Kirchhoff et al. 2010). Insofern ist der Einfluss dieser Komplikationen bei Patienten mit schwerer akuter nekrotisierender Pankreatitis und den hiermit assoziierten systemischen Komplikationen und per se deutlich erhöhter Morbiditätsrate ein relevanter Risikofaktor für das Resultat des Patienten. Postoperative Komplikationen können bis zu einer 4-fachen Verlängerung der Aufenthaltsdauer auf der Intensivstation führen. Wie in den USA errechnet wurde, zieht allein jede pulmonale Komplikation Extrakosten von 52.000 US-Dollar nach sich (Siewert 2009). Dies wirft die Frage auf, ob komplikationsgefährdete Patienten erkennbar wären und somit gar nicht erst der Gefährdung eines solchen Eingriffs ausgesetzt werden sollten. Das American College of Surgery veröffentlichte dazu 2009 ein Modell zur besseren präoperativen Risikokalkulierung der Patienten, das hierzulande jedoch noch nicht in die tägliche klinische Routine integriert ist (Cohen et al. 2009).

Ferner stellen Langzeitprobleme wie Narbenhernien, Fisteln oder das lebenslange Vorhandensein von Enterostomien nicht nur physische, sondern auch psychische Belastungen für die Betroffenen und auch für deren Angehörige dar (Murray et al. 2011, Schloricke et al. 2012).

So stellt sich die Frage, inwiefern lebensverlängernde Maßnahmen in Form eines radikal chirurgischen Vorgehens die Lebensqualität der Patienten so weit einschränken, dass aus ethischer Sicht von einem solchen Eingriff abzuraten wäre. Dem sicherlich gegenüberzustellen ist, dass - vor dem Hintergrund des erklärten Patientenwillens - lebensrettende Maßnahmen in jedem Falle als prioritär angesehen werden. 
Trotz eventuell nachfolgender Einschränkung in der Lebensqualität der Patienten ist das primäre Ziel das Überleben des Patienten. Der Erfolg einer Therapie der komplizierten akuten nekrotisierenden Pankreatitis wird, wie letztlich bei jeder Therapie, primär am Überleben der Patienten gemessen. Die Frage der Ethik rückt hierbei in den Hintergrund (Winter Gasparoto et al. 2015).

Die Krankenhausverweildauer lag im Gesamtkollektiv durchschnittlich bei etwa 70 Tagen, wobei die Verweildauer der Patienten auf der Intensivstation etwa Zweidrittel der Zeit davon ausmachte.

Beim Vergleich der Teilkollektive zeigte sich, dass die Homburger Patienten im Durchschnitt circa zwei Wochen länger im Krankenhaus blieben als die Göttinger Patienten. Dieses Ergebnis kann sicherlich mit dem chirurgischen Therapiekonzept des jeweiligen Universitätsklinikums in Zusammenhang gesehen werden. Denn wie weiterhin im ersten Subgruppenvergleich demonstriert werden konnte, lag die Krankenhausverweildauer derer, die einer Kolektomie unterzogen worden waren, 25 Tage höher als bei Patienten ohne Eingriff am Kolon. Bei isolierter Analyse der überlebenden Patienten fiel das Ergebnis noch deutlicher aus. Die Krankenhausverweildauer der kolektomierten Patienten war hier fast doppelt so hoch wie die der nicht kolektomierten Patienten. Die isolierte Auswertung der Patienten, die innerhalb ihres Krankenhausaufenthaltes verstarben, zeigte ebenfalls signifikante Unterschiede. Kolektomierte Patienten verstarben durchschnittlich nach etwa 4 Wochen, Patienten ohne Koloneingriff überlebten doppelt so lange und Patienten mit Kolonteilresektion wiesen mit ungefähr 24 Wochen die weitaus längste Krankenhausverweildauer bzw. Überlebenszeit im Krankenhaus auf. Es wurde letzten Endes also deutlich, dass die Krankenhausverweildauer der Patienten ohne Kolektomie etwa bei 60 Tagen lag unabhängig davon, ob sie überlebten oder während des Krankenhausaufenthaltes verstarben. Die kolektomierten Patienten, die verstarben, taten dies bereits nach 27 Tagen, während diejenigen Patienten, die die Erkrankung überlebten, mit 116 Tagen die weitaus längste Krankenhausverweildauer zeigten.

Diese Zahlen können den Effekt der Kolektomie in Bezug auf die Krankenhausverweildauer letztlich nicht belegen. Aufgrund der zu geringen Fallzahlen ist eine Unterscheidung, ob die Patienten, die kolektomiert werden mussten, tatsächlich infolge der Kolektomie einen verlängerten Krankenhausaufenthalt hatten, und ob die erhöhte Anzahl an Re-Operationen ebenfalls der Kolektomie geschuldet war, nicht möglich. Ebenso ist durchaus denkbar, dass nur die sehr kritisch erkrankten Patienten mit ausgedehntesten Nekrosen und Arrosion des Mesocolon transversum oder gar des Kolons selbst einer Kolektomie unterzogen wurden, um eine effektive Sanierung des die Kontamination der Nekrosen unterhaltenden Fokus zu erzielen. Somit wäre aufgrund der schlechteren Ausgangslage des jeweils betroffenen Patienten per se ein längerer intensivmedizinischer Therapiebedarf und komplikationsträchtiger Verlauf denkbar. Auch wenn die vorliegenden retrospektiv erhobenen Daten 
diese These nicht beweisen können, so muss doch retrospektiv aufgrund der zur Verfügung stehenden OP-Berichte angenommen werden, dass insbesondere diejenigen Patienten kolektomiert wurden, die maximal kritisch erkrankt waren und bei denen eine radikale und effektive Sanierung angestrebt war. Aufgrund der schlechten Ausgangslage und der Schwere der Erkrankung der Patienten sind die verlängerte Krankenhaus- und Intensivverweildauer eher vor diesem Hintergrund als vor dem Hintergrund der Kolektomie zu sehen.

In Anbetracht der beobachteten Unterschiede gilt es, auch gesundheitsökonomische Faktoren in die Diskussion mit einzubringen. So führen verlängerte Krankenhausaufenthalte, insbesondere auf der Intensivstation, zu einem massiven Anstieg der Behandlungskosten. Die Intensivmedizin verursacht bei einem Anteil von $5 \%$ der Krankenhausbetten einen Anteil von $20 \%$ an den Krankenhauskosten (Martin 1998). Große Erfolge auf dem Gebiet der Intensivmedizin haben in den letzten Jahren dazu geführt, dass immer mehr Patienten ausgedehnten operativen Eingriffen unterzogen werden können, welche jedoch häufig mit einem langwierigen Aufenthalt auf einer Intensivstation verbunden sind. Die Frage der langfristigen Finanzierbarkeit dieses Fortschritts ist momentan Gegenstand vieler Diskussionen.

Die Subgruppenanalysen, die den Stellenwert des Zeitpunktes der Kolektomie untersuchten, zeigten eine Tendenz zugunsten eines verbesserten Ergebnisses der Patienten, sofern die Kolektomie frühzeitig durchgeführt wurde. Aufgrund zu geringer Fallzahlen erreichen die Unterschiede dieser Analyse jedoch keine statistische Signifikanz und sind nicht als sehr robust anzusehen. Dennoch kann aufgrund der vorliegenden Daten spekuliert werden, dass durch eine frühzeitige Kolektomie, welche idealerweise im Rahmen des Ersteingriffs durchgeführt wird, die Pathophysiologie der komplizierten akuten nekrotisierenden Pankreatitis durchbrochen werden kann. Dies spiegeln die deutlich reduzierten Krankenaus- sowie 30- und 90-Tagesmortalitäten wider. Weiterhin konnte bei den frühzeitig kolektomierten Patienten eine deutliche Reduktion der Gesamtverweildauer im Krankenhaus sowie auf Intensivstation beobachtet werden. Somit geben die Daten einen ersten Hinweis, dass die chirurgische Entfernung der die bakterielle Superinfektion der Nekrosen unterhaltenden Quelle durch eine Kolektomie für den betroffenen Patienten vorteilhaft sein könnte. Auf pathophysiologischer Ebene könnte die Kolektomie zu einer Verhinderung einer fortgesetzten Superinfektion der Pankreasnekrosen sowie der persistierenden Endotoxinämie führen. Folge der durchbrochenen Endotoxinämie wären im Wesentlichen eine Unterbrechung der systemischen Hyperinflammation sowie eine Stabilisierung der Mikrozirkulation, welche im Rahmen einer Sepsis bekanntermaßen massiv gestört ist (De Backer et al. 2014, Hernandez et al. 2013, Lundy und Trzeciak 2011). Die aus der rekonstituierten Mikrozirkulation resultierende suffiziente Oxygenierung des Gewebes und die wiederhergestellte intrazelluläre Sauerstoffutilisation verhindern das Entstehen weiterer Pankreasnekrosen und führen systemisch zu 
einer Stabilisierung der septischen kardiozirkulatorischen und pulmonalen Insuffizienz, die die häufigsten sekundären Organkomplikationen des vorliegenden Kollektivs darstellten (Cohen und Hollenberg 2001, Gutierrez et al. 1989, Morisaki und Sibbald 2004).

Eine abschließende Auswertung, in der zentrumsübergreifend der Stellenwert der Kolektomie dargestellt werden sollte, ergab, dass die kolektomierten Homburger Patienten die niedrigste Krankenhausmortalität aufwiesen (33 \%). Die Homburger Patienten ohne Kolektomie zeigten hingegen ähnlich den Göttinger Patienten, von denen ebenfalls kein Patient kolektomiert worden war, eine Krankenhausmortalität von etwa $53 \%$ Ob in Homburg die Ausgangslage der Patienten die Entscheidungsfindung einer zusätzlichen Kolektomie beeinflusste und eventuell schwerer Erkrankten dieser zusätzliche Eingriff einer Kolektomie nicht zugemutet wurde, lässt sich aufgrund der verfügbaren Daten nicht evaluieren. Ebenso wäre es denkbar, dass Patienten mit einer Kolektomie ein anderes postoperatives Intensivmanagement zuteil wurde, welches das veränderte Outcome erklären könnte.

Einen weiteren Diskussionspunkt stellt die Vergleichbarkeit der beiden Teilkollektive, Homburg/Saar und Göttingen, dar. Beide Teilkollektive wurden in verschiedenen Zeiträumen therapiert. Während in Homburg auf Patientendaten im Zeitraum von 2002 bis 2012 zurückgegriffen werden konnte, wurden in Göttingen nur Patientendaten des Zeitraumes von 2008 bis 2012 analysiert. Dies beinhaltet die Möglichkeit, dass sich im Homburger Kollektiv auch Patienten befinden, die nach älteren Standards der Intensivtherapie behandelt wurden. Gerade das intensivmedizinische Management der letzten Jahre hat mit der Etablierung differenzierter Beatmungskonzepte, der Entwicklung neuer anti-infektiver Substanzen und moderner Sepsis-Therapiealgorithmen enorme Fortschritte erzielt, sodass aufgrund des langen Rekrutierungszeitraums im Homburger Kollektiv eine interne Inhomogenität bestehen könnte.

Bei der Interpretation der Subgruppenanalysen der verschiedenen Therapieoptionen ist zudem als kritisch anzumerken, dass es sich bei den Vergleichskollektiven um unterschiedlich große Fallzahlen handelte. Aufgrund der insgesamt geringen Fallzahlen, insbesondere der kolektomierten Patienten, gestaltete sich eine statistisch robuste Beurteilung der Kolektomie und deren Einfluss auf das Outcome der Patienten als außerordentlich schwierig und eine Interpretation der Ergebnisse als wenig aussagekräftig. Alleine um bei dem beobachteten Unterschied der Krankenhausmortalität eine statistische Power von 0,8 zu erzielen, wäre ein Kollektiv von über 100 Patienten erforderlich. Aufgrund der sehr engen Einschlusskriterien war ein solches Kollektiv im Rahmen der aktuellen Studie jedoch retrospektiv nicht zu rekrutieren. Eine weitere methodologische Einschränkung der Studie ist die Tatsache, dass das Konzept der Kolektomie in Göttingen nicht etabliert ist. Es bestand somit keine 
Möglichkeit, das Therapiekonzept der zusätzlichen Kolektomie bei akuter nekrotisierender Pankreatitis zentrumsübergreifend miteinander zu vergleichen, sondern lediglich einen Vergleich der beiden Zentren und der jeweilig dort etablierten Therapiekonzepte vorzunehmen.

Da es sich um eine retrospektive Auswertung digitalisierter bzw. papierbasierter Patientenakten handelt, ergeben sich einige weitere Einschränkungen bezüglich der Aussagekraft der Ergebnisse. Retrospektive Analysen können nur Aussagen mit einem geringen Evidenzgrad treffen, da Selektionsfehler der Patienten zu Verzerrungen des Studienresultates führen können. Um schlussendlich die Frage eines möglichen Benefits für Patienten mit akuter nekrotisierender Pankreatitis durch eine zusätzliche Kolektomie endgültig zu klären, bedarf es einer kontrolliert durchgeführten randomisierten prospektiven Studie. Diese ist jedoch aufgrund der nur sehr geringen Fallzahlen an Patienten mit einer komplizierten akuten nekrotisierenden Pankreatitis sowie der methodologisch erforderlichen ethischen Implikationen, d.h. einem Patienten gegebenenfalls eine erforderliche Kolektomie aus Studiengründen vorzuenthalten, nicht realisierbar. Erschwerend kommt hinzu, dass die Qualität einer retrospektiven Analyse sehr stark von der Gewissenhaftigkeit und Ausführlichkeit der Aktendokumentation sowie der korrekten Datenaufbewahrung und -verfügbarkeit abhängig ist. So ist eine retrospektive Datenanalyse nur so gut wie die Dokumentation, auf die sie gestützt ist. Eine Überprüfung der Richtigkeit ist im Nachhinein nicht möglich. Überdies wäre eine umfangreichere Dokumentation bei der Erfassung von Komplikationen und Organersatzverfahren vorteilhaft gewesen. So konnte bei der Datenerhebung nicht differenziert werden, ob es sich um eine sekundäre Organkomplikation der akuten nekrotisierenden Pankreatitis oder um eine Komplikation der Erkrankung selbst oder gar um eine Folgekomplikation der Operation handelte. Zudem war die uns vorliegende Datendokumentation einiger Parameter wie beispielsweise der ätiologischen Faktoren oder auch der postoperativen Komplikationen lückenhaft, was die Aussagekraft dieser Parameter weiterhin schmälert.

Zusammenfassend lässt sich somit sagen, dass die vorliegenden Daten eine tendenzielle, aber statistisch nicht signifikant niedrigere Mortalität bei Patienten mit einer komplizierten akuten nekrotisierenden Pankreatitis, welche im Rahmen ihrer Behandlung einer Kolektomie zugeführt wurden, demonstrierten. Insbesondere eine frühzeitig durchgeführte Kolektomie scheint für die betroffenen Patienten im Hinblick auf das Mortalitätsrisiko sowie die Verweildauern auf Intensivstation und im Krankenhaus von Vorteil zu sein.

Das zur Verfügung stehende Kollektiv ist jedoch zu klein, um eine generelle Empfehlung daraus abzuleiten, dass durch eine zusätzliche Resektion des Kolons bei der Therapie der akuten nekrotisierenden Pankreatitis das Outcome der Patienten positiv beeinflusst werden kann. 
Nichtsdestoweniger können Kolektomien im Einzelfall als Individualentscheidungen erforderlich werden, wobei aufgrund der vorliegenden Daten in diesem Fall eine möglichst frühzeitige Kolektomie, idealerweise im Rahmen des ersten stattfindenden chirurgischen Eingriffs, zu favorisieren wäre. 


\section{$5 \quad$ Zusammenfassung}

Die akute nekrotisierende Pankreatitis ist ein Krankheitsbild mit steigender Inzidenz. Trotz Verbesserungen in Diagnostik, intensivmedizinischer Therapie sowie fortgeschrittener chirurgischer Techniken in den letzten Jahrzehnten bleibt die Letalität hoch. Insbesondere die Superinfektion der Nekrosen mit konsekutiv systemischen Komplikationen führt zu sekundären Organschäden bis hin zum Multiorganversagen. Als Quelle der bakteriellen Kontamination wird das Kolon angesehen.

Ziel dieser Studie war es, retrospektiv unterschiedliche Konzepte des chirurgischen Managements der akuten nekrotisierenden Pankreatitis an zwei deutschen Universitätsklinika, dem Universitätsklinikum des Saarlandes sowie der Universitätsmedizin Göttingen, miteinander zu vergleichen. Dabei wurden anamnestische und perioperative Daten derjenigen Patienten, die im Zeitraum 2002 bis 2012 aufgrund einer komplizierten akuten nekrotisierenden Pankreatitis therapiert wurden und einer operativen und intensivmedizinischen Behandlung bedurften, erfasst und bezüglich der operativen Therapie und objektiver Parameter analysiert.

Insgesamt wurden 56 Patienten in die Analyse eingeschlossen. Das Durchschnittsalter betrug 57,2 $\pm 14,9$ Jahre. Es bestand kein relevanter Unterschied zwischen den Kollektiven aus Homburg/Saar und Göttingen. Die Pankreatitiden waren im Wesentlichen biliär, post-interventionell und äthyltoxisch bedingt. Die Krankenhausmortalität im Gesamtkollektiv betrug 50 \%, die 30- und 90-Tages-Mortalitäten betrugen $21,4 \%$ bzw. $35,7 \%$ bei einer Gesamtverweildauer von 70,5 $\pm 57,0$ Tagen. Die Subgruppenanalysen zeigten, dass Patienten nach Kolektomie $(n=9)$ eine deutlich reduzierte Krankenhaus-Mortalität im Vergleich zu nicht-kolektomierten Patienten ( $n=47$ ) aufwiesen (33 \% vs. 53,2 $\%$ ), wobei die Krankenhausverweil- und Intensivverweildauern kolektomierter Patienten länger waren ( $86,6 \pm 54,3$ d vs. $67,3 \pm 57,6$ d, bzw. $52,9 \pm 23,7$ d vs. $45,6 \pm 49,3$ d). Die Durchführung der Kolektomie im Rahmen des ersten erforderlichen chirurgischen Eingriffs führte zu einer deutlichen Reduktion der Krankenhaus- bzw. 30-Tages- und 90-Tages-Mortalität. Der Zeitpunkt der Kolektomie nach stationärer Aufnahme bzw. nach Diagnosestellung hingegen war für das Resultat der Patienten nicht entscheidend. Bei Vergleich der nicht-kolektomierten Patienten an beiden Zentren konnten keine Unterschiede bezüglich der Krankenhaus-, 30-Tages- und 90-Tages-Mortalitäten sowie der Gesamt- und Intensivverweildauern gezeigt werden.

Aus den Ergebnissen dieser Studie lässt sich abschließend sagen, dass für Patienten mit komplizierter akuter nekrotisierender Pankreatitis ein möglicher Benefit durch eine frühzeitige Kolektomie gezeigt werden konnte. Diese Ergebnisse verfügen jedoch aufgrund der geringen Fallzahlen über eine geringe statistische Aussagekraft und dürfen daher nicht als endgültige Therapieempfehlung aufgefasst werden. 


\section{$6 \quad$ Literaturverzeichnis}

Abou-Assi S, Craig K, O'Keefe SJ (2002): Hypocaloric jejunal feeding is better than total parenteral nutrition in acute pancreatitis: results of a randomized comparative study. Am J Gastroenterol 97, 2255-2262

Adams DB, Davis BR, Anderson MC (1994): Colonic complications of pancreatitis. The American surgeon $60,44-49$

Amano H, Takada T, Isaji S, Takeyama Y, Hirata K, Yoshida M, Mayumi T, Yamanouchi E, Gabata T, Kadoya M (2010): Therapeutic intervention and surgery of acute pancreatitis. J Hepatobiliary Pancreat Sci 17, 53-59

Amelsberg A, Fölsch UR (1997): Komplikationen bei der endoskopischen Papillotomie. Z Gastroenterol $\underline{35}, 1111-111435$

Ando T, Tsuyuguchi T, Okugawa T, Saito M, Ishihara T, Yamaguchi T, Saisho H (2003): Risk factors for recurrent bile duct stones after endoscopic papillotomy. Gut $\underline{52}, 116-121$

Arvanitakis M, Delhaye M, De Maertelaere V, Bali M, Winant C, Coppens E, Jeanmart J, Zalcman M, Van Gansbeke D, Deviere J (2004): Computed tomography and magnetic resonance imaging in the assessment of acute pancreatitis. Gastroenterology $\underline{126}, 715-723$

Bakker OJ, van Santvoort HC, van Brunschot S, Geskus RB, Besselink MG, Bollen TL, van Eijck CH, Fockens P, Hazebroek EJ, Nijmeijer RM (2012): Endoscopic transgastric vs surgical necrosectomy for infected necrotizing pancreatitis: a randomized trial. JAMA 307, 1053-1061

Balthazar EJ (2002): Complications of acute pancreatitis: clinical and CT evaluation. Radiol Clin North Am 40, 1211-1227

Bank S, Singh P, Pooran N, Stark B (2002): Evaluation of factors that have reduced mortality from acute pancreatitis over the past 20 years. J Clin Gastroenterol 35, 50-60

Banks PA, Freeman ML, Practice Parameters Committee of the American College of G (2006): Practice guidelines in acute pancreatitis. Am J Gastroenterol 101, 2379-2400

Banks PA, Bollen TL, Dervenis C, Gooszen HG, Johnson CD, Sarr MG, Tsiotos GG, Vege SS, Acute Pancreatitis Classification Working G (2013): Classification of acute pancreatitis--2012: revision of the Atlanta classification and definitions by international consensus. Gut $\underline{62}, 102-111$

Bausch D, Wellner U, Kahl S, Kuesters S, Richter-Schrag HJ, Utzolino S, Hopt UT, Keck T, Fischer A (2012): Minimally invasive operations for acute necrotizing pancreatitis: comparison of minimally invasive retroperitoneal necrosectomy with endoscopic transgastric necrosectomy. Surgery 152, S128-134

Beger HG, Bittner R, Block S, Buchler M (1986): Bacterial contamination of pancreatic necrosis. A prospective clinical study. Gastroenterology $\underline{91}, 433-438$ 
Beger HG, Büchler M, Bittner R, Block S, Nevalainen T, Roscher R (1988): Necrosectomy and postoperative local lavage in necrotizing pancreatitis. Br. J. Surg. 75, 207-212

Beger HG, Rau B, Mayer J, Pralle U (1997): Natural course of acute pancreatitis. World J Surg 21, 130135

Bengmark S (1998): Ecological control of the gastrointestinal tract. The role of probiotic flora. Gut 42 , 27

Besselink MG, van Santvoort HC, Buskens E, Boermeester MA, van Goor H, Timmerman HM, Nieuwenhuijs VB, Bollen TL, van Ramshorst B, Witteman BJ (2008): Probiotic prophylaxis in predicted severe acute pancreatitis: a randomised, double-blind, placebo-controlled trial. Lancet $371,651-659$

Besselink MG, van Santvoort HC, Boermeester MA, Nieuwenhuijs VB, van Goor H, Dejong CH, Schaapherder AF, Gooszen HG, Dutch Acute Pancreatitis Study G (2009): Timing and impact of infections in acute pancreatitis. Br J Surg 96, 267-273

Besselink MG, van Santvoort HC, Freeman M, Gardner T (2013): IAP/APA evidence-based guidelines for the management of acute pancreatitis. Pancreatology 13 , e1-15

Bradley EL, 3rd (1993): A clinically based classification system for acute pancreatitis. Summary of the International Symposium on Acute Pancreatitis, Atlanta, Ga, September 11 through 13, 1992. Arch Surg 128, 586-590

Brown A, Orav J, Banks PA (2000): Hemoconcentration is an early marker for organ failure and necrotizing pancreatitis. Pancreas $20,367-372$

Brown A, Baillargeon JD, Hughes MD, Banks PA (2002): Can fluid resuscitation prevent pancreatic necrosis in severe acute pancreatitis? Pancreatology $2,104-107$

Büchler MW, Gloor B, Muller CA, Friess H, Seiler CA, Uhl W (2000): Acute necrotizing pancreatitis: treatment strategy according to the status of infection. Ann Surg 232, 619-626

Cohen ES, Hollenberg SM (2001): Tissue oxygenation and sepsis. Crit Care Med 29, 1479-1480

Cohen ME, Bilimoria KY, Ko CY, Hall BL (2009): Development of an American College of Surgeons National Surgery Quality Improvement Program: morbidity and mortality risk calculator for colorectal surgery. J Am Coll Surg 208, 1009-1016

Connor S, Alexakis N, Raraty MG, Ghaneh P, Evans J, Hughes M, Garvey CJ, Sutton R, Neoptolemos JP (2005): Early and late complications after pancreatic necrosectomy. Surgery 137, 499-505

Daams F, Luyer M, Lange JF (2013): Colorectal anastomotic leakage: aspects of prevention, detection and treatment. World J Gastroenterol 19, 2293-2297

De Backer D, Orbegozo Cortes D, Donadello K, Vincent JL (2014): Pathophysiology of microcirculatory dysfunction and the pathogenesis of septic shock. Virulence $\underline{5}, 73-79$

Dervenis C, Johnson CD, Bassi C, Bradley E, Imrie CW, McMahon MJ, Modlin I (1999): Diagnosis, objective assessment of severity, and management of acute pancreatitis. Santorini consensus conference. Int J Pancreatol 25, 195-210 
Doberneck RC (1989): Intestinal fistula complicating necrotizing pancreatitis. Am J Surg 158, 581-583; discussion 583-584

Eatock FC, Chong P, Menezes N, Murray L, McKay CJ, Carter CR, Imrie CW (2005): A randomized study of early nasogastric versus nasojejunal feeding in severe acute pancreatitis. Am J Gastroenterol 100, 432-439

Fagenholz PJ, Fernandez-del Castillo C, Harris NS, Pelletier AJ, Camargo CA, Jr. (2007): Direct medical costs of acute pancreatitis hospitalizations in the United States. Pancreas $35,302-307$

Fernandez-del Castillo C, Rattner DW, Makary MA, Mostafavi A, McGrath D, Warshaw AL (1998): Debridement and closed packing for the treatment of necrotizing pancreatitis. Ann Surg $\underline{228}$, 676-684

Forsmark CE, Baillie J, Practice AGAIC, Economics C, Board AGAIG (2007): AGA Institute technical review on acute pancreatitis. Gastroenterology 132, 2022-2044

Fritz S, Hackert T, Hartwig W, Rossmanith F, Strobel O, Schneider L, Will-Schweiger K, Kommerell M, Buchler MW, Werner J (2010): Bacterial translocation and infected pancreatic necrosis in acute necrotizing pancreatitis derives from small bowel rather than from colon. Am J Surg 200, 111117

Garg PK, Tandon RK, Madan K (2007): Is biliary microlithiasis a significant cause of idiopathic recurrent acute pancreatitis? A long-term follow-up study. Clin Gastroenterol Hepatol $\underline{5}, 75-79$

Guarner F, Malagelada JR (2003): Gut flora in health and disease. Lancet 361, 512-519

Gumaste VV, Roditis N, Mehta D, Dave PB (1993): Serum lipase levels in nonpancreatic abdominal pain versus acute pancreatitis. Am J Gastroenterol 88, 2051-2055

Gupta K, Wu B (2010): In the clinic. Acute pancreatitis. Ann Intern Med 153, 51-55; 516

Gurusamy KS, Farouk M, Tweedie JH (2005): UK guidelines for management of acute pancreatitis: is it time to change? Gut $\underline{54}, 1344-1345$

Gutierrez G, Lund N, Bryan-Brown CW (1989): Cellular oxygen utilization during multiple organ failure. Crit Care Clin $\underline{5}, 271-287$

Gutierrez-Jimenez AA, Castro-Jimenez E, Lagunes-Cordoba R (2014): [Total serum calcium and corrected calcium as severity predictors in acute pancreatitis]. Rev Gastroenterol Mex $\underline{79}, 13-21$

Hernandez G, Bruhn A, Ince C (2013): Microcirculation in sepsis: new perspectives. Curr Vasc Pharmacol 11, 161-169

Isaji S, Takada T, Kawarada Y, Hirata K, Mayumi T, Yoshida M, Sekimoto M, Hirota M, Kimura Y, Takeda K (2006): JPN Guidelines for the management of acute pancreatitis: surgical management. J Hepatobiliary Pancreat Surg 13, 48-55

Isenmann R, Beger HG (1999): Natural history of acute pancreatitis and the role of infection. Baillieres Best Pract Res Clin Gastroenterol 13, 291-301 
Isenmann R, Schwarz M, Rau B, Trautmann M, Schober W, Beger HG (2002): Characteristics of infection with Candida species in patients with necrotizing pancreatitis. World J Surg 26, 372376

Jakobs R, Adamek MU, von Bubnoff AC, Riemann JF (2000): Buprenorphine or procaine for pain relief in acute pancreatitis. A prospective randomized study. Scand J Gastroenterol 35, 1319-1323

Johnson CD, Kingsnorth AN, Imrie CW, McMahon MJ, Neoptolemos JP, McKay C, Toh SK, Skaife P, Leeder PC, Wilson P (2001): Double blind, randomised, placebo controlled study of a platelet activating factor antagonist, lexipafant, in the treatment and prevention of organ failure in predicted severe acute pancreatitis. Gut $\underline{48}, 62-69$

Kahl S, Zimmermann S, Pross M, Schulz HU, Schmidt U, Malfertheiner P (2004): Procaine hydrochloride fails to relieve pain in patients with acute pancreatitis. Digestion $\underline{69}, 5-9$

Khanna AK, Meher S, Prakash S, Tiwary SK, Singh U, Srivastava A, Dixit VK (2013): Comparison of Ranson, Glasgow, MOSS, SIRS, BISAP, APACHE-II, CTSI Scores, IL-6, CRP, and Procalcitonin in Predicting Severity, Organ Failure, Pancreatic Necrosis, and Mortality in Acute Pancreatitis. HPB Surg 2013, 367581

Kimura Y, Takada T, Kawarada Y, Hirata K, Mayumi T, Yoshida M, Sekimoto M, Hirota M, Takeda K, Isaji S (2006): JPN Guidelines for the management of acute pancreatitis: treatment of gallstoneinduced acute pancreatitis. J Hepatobiliary Pancreat Surg 13, 56-60

Kirchhoff P, Clavien PA, Hahnloser D (2010): Complications in colorectal surgery: risk factors and preventive strategies. Patient Saf Surg $\underline{4}, 5$

Kriwanek S, Armbruster C, Beckerhinn P, Dittrich K (1994): Prognostic factors for survival in colonic perforation. Int J Colorectal Dis $\underline{9}, 158-162$

Kriwanek S, Armbruster C, Beckerhinn P, Dittrich K, Redl E (1996): Improved results after aggressive treatment of colonic involvement in necrotizing pancreatitis. Hepatogastroenterology $\underline{43}, 1627-$ 1632

Kriwanek S, Gschwantler M, Beckerhinn P, Armbruster C, Roka R (1999): Complications after surgery for necrotising pancreatitis: risk factors and prognosis. Eur J Surg 165, 952-957

Kylanpaa-Back ML, Takala A, Kemppainen E, Puolakkainen P, Haapiainen R, Repo H (2001): Procalcitonin strip test in the early detection of severe acute pancreatitis. Br J Surg 88, 222-227

Lankisch PG, Burchard-Reckert S, Lehnick D (1999): Underestimation of acute pancreatitis: patients with only a small increase in amylase/lipase levels can also have or develop severe acute pancreatitis. Gut $44,542-544$

Lankisch PG, Assmus C, Lehnick D, Maisonneuve P, Lowenfels AB (2001a): Acute pancreatitis: does gender matter? Dig Dis Sci 46, 2470-2474

Lankisch PG, Blum T, Bruns A, Droge M, Brinkmann G, Struckmann K, Nauck M, Maisonneuve P, Lowenfels $A B$ (2001b): Has blood glucose level measured on admission to hospital in a patient with acute pancreatitis any prognostic value? Pancreatology 1, 224-229

Lankisch PG, Assmus C, Maisonneuve P, Lowenfels AB (2002): Epidemiology of pancreatic diseases in Luneburg County. A study in a defined german population. Pancreatology 2, 469-477 
Lukash WM (1967): Complications of acute pancreatitis. Unusual sequelae in 100 cases. Arch Surg 94, 848-852

Lundy DJ, Trzeciak S (2011): Microcirculatory dysfunction in sepsis. Crit Care Nurs Clin North Am 23, 67-77

Martin E (1998): Sind Fortschritte in der Intensivmedizin noch finanzierbar. J Anästh 2, 1-9

Menger MD, Plusczyk T, Vollmar B (2001): Microcirculatory derangements in acute pancreatitis. J Hepatobiliary Pancreat Surg $\underline{8}, 187-194$

Mier J, Leon EL, Castillo A, Robledo F, Blanco R (1997): Early versus late necrosectomy in severe necrotizing pancreatitis. Am J Surg 173, 71-75

Mitchell RM, Byrne MF, Baillie J (2003): Pancreatitis. Lancet 361, 1447-1455

Morisaki H, Sibbald WJ (2004): Tissue oxygen delivery and the microcirculation. Crit Care Clin 20, 213223

Mossner J (2011): Update lecture: benign diseases of the exocrine pancreas. Dig Dis 29 Suppl 1, 9-16

Muller CA, Uhl W, Printzen G, Gloor B, Bischofberger H, Tcholakov O, Buchler MW (2000): Role of procalcitonin and granulocyte colony stimulating factor in the early prediction of infected necrosis in severe acute pancreatitis. Gut $\underline{46}, 233-238$

Munoz-Bongrand N, Panis Y, Soyer P, Riche F, Laisne MJ, Boudiaf M, Valleur P (2001): Serial computed tomography is rarely necessary in patients with acute pancreatitis: a prospective study in 102 patients. J Am Coll Surg 193, 146-152

Murray BW, Cipher DJ, Pham T, Anthony T (2011): The impact of surgical site infection on the development of incisional hernia and small bowel obstruction in colorectal surgery. Am J Surg 202, 558-560

Nagpal AP, Soni H, Haribhakti S (2015): Severe Colonic Complications requiring Sub-Total Colectomy in Acute Necrotizing Pancreatitis-A Retrospective Study of 8 Patients. Indian J Surg 77, 3-6

Nealon WH, Bawduniak J, Walser EM (2004): Appropriate timing of cholecystectomy in patients who present with moderate to severe gallstone-associated acute pancreatitis with peripancreatic fluid collections. Ann Surg 239, 741-749; discussion 749-751

Ney MV, Maluf-Filho F, Sakai P, Zilberstein B, Gama-Rodrigues J, Rosa H (2005): Echo-endoscopy versus endoscopic retrograde cholangiography for the diagnosis of choledocholithiasis: the influence of the size of the stone and diameter of the common bile duct. Arq Gastroenterol $\underline{42}$, 239-243

Paye F, Rotman N, Radier C, Nouira R, Fagniez PL (1998): Percutaneous aspiration for bacteriological studies in patients with necrotizing pancreatitis. Br J Surg $\underline{85}, 755-759$

Petrov MS, Uchugina AF, Kukosh MV (2008): Does endoscopic retrograde cholangiopancreatography reduce the risk of local pancreatic complications in acute pancreatitis? A systematic review and metaanalysis. Surg Endosc 22, 2338-2343 
Ranson JH (1982): Etiological and prognostic factors in human acute pancreatitis: a review. Am J Gastroenterol 77, 633-638

Rau B, Steinbach G, Gansauge F, Mayer JM, Grunert A, Beger HG (1997): The potential role of procalcitonin and interleukin 8 in the prediction of infected necrosis in acute pancreatitis. Gut $\underline{41}, 832-840$

Rau B, Poch B, Gansauge F, Bauer A, Nussler AK, Nevalainen T, Schoenberg MH, Beger HG (2000): Pathophysiologic role of oxygen free radicals in acute pancreatitis: initiating event or mediator of tissue damage? Ann Surg 231, 352-360

Runzi M, Layer P, Buchler MW, Beger HG, Ell C, Folsch UR, Goebell H, Hopt UT, Lankisch PG, Schmidt WE (2000): [The therapy of acute pancreatitis. General guidelines. Working group of the Society for Scientific-Medical Specialties]. Z Gastroenterol 38, 571-581

Runzi M, Niebel W, Goebell H, Gerken G, Layer P (2005): Severe acute pancreatitis: nonsurgical treatment of infected necroses. Pancreas 30, 195-199

Russell JC, Welch JP, Clark DG (1983): Colonic complications of acute pancreatitis and pancreatic abscess. Am J Surg 146, 558-564

Sarr MG (2013): 2012 revision of the Atlanta classification of acute pancreatitis. Pol Arch Med Wewn $123,118-124$

Schloricke E, Zimmermann M, Hoffmann M, Laubert T, Nolde J, Hildebrand P, Bruch HP, Bouchard R (2012): [Surgical treatment and prognosis of rectovaginal fistulae according to their origin]. Zentralbl Chir 137, 390-395

Shaheen NJ, Hansen RA, Morgan DR, Gangarosa LM, Ringel Y, Thiny MT, Russo MW, Sandler RS (2006): The burden of gastrointestinal and liver diseases, 2006. Am J Gastroenterol 101, 21282138

Sharma VK, Howden CW (1999): Metaanalysis of randomized controlled trials of endoscopic retrograde cholangiography and endoscopic sphincterotomy for the treatment of acute biliary pancreatitis. Am J Gastroenterol 94, 3211-3214

Sharma VK, Howden CW (2001): Prophylactic antibiotic administration reduces sepsis and mortality in acute necrotizing pancreatitis: a meta-analysis. Pancreas $\underline{22}, 28-31$

Siewert JR (2009): [It is not the postoperative complication which is the problem, but how to deal with it!]. Chirurg $\underline{80,}, 767$

Smith RL, Bohl JK, McElearney ST, Friel CM, Barclay MM, Sawyer RG, Foley EF (2004): Wound infection after elective colorectal resection. Ann Surg 239, 599-605; discussion 605-597

Spanier BW, Dijkgraaf MG, Bruno MJ (2008): Epidemiology, aetiology and outcome of acute and chronic pancreatitis: An update. Best Pract Res Clin Gastroenterol 22, 45-63

Staritz M (1988): Pharmacology of the sphincter of Oddi. Endoscopy 20 Suppl 1, 171-174

Tenner S, Dubner H, Steinberg W (1994): Predicting gallstone pancreatitis with laboratory parameters: a meta-analysis. Am J Gastroenterol 89, 1863-1866 
Tenner S, Baillie J, DeWitt J, Vege SS, American College of G (2013): American College of Gastroenterology guideline: management of acute pancreatitis. Am J Gastroenterol 108, 1400$1415 ; 1416$

Uhl W, Warshaw A, Imrie C, Bassi C, McKay CJ, Lankisch PG, Carter R, Di Magno E, Banks PA, Whitcomb DC (2002): IAP Guidelines for the Surgical Management of Acute Pancreatitis. Pancreatology 2, 565-573

Van Minnen LP, Besselink MGH, Bosscha K, Van Leeuwen MS, Schipper MEI, Gooszen HG (2004): Colonic involvement in acute pancreatitis. A retrospective study of 16 patients. Digestive surgery 21, 33-38; discussion 39-40 \% U

van Santvoort HC, Besselink MG, Bakker OJ, Hofker HS, Boermeester MA, Dejong CH, van Goor H, Schaapherder AF, van Eijck CH, Bollen TL (2010): A step-up approach or open necrosectomy for necrotizing pancreatitis. N Engl J Med 362, 1491-1502

Venneman NG, Buskens E, Besselink MG, Stads S, Go PM, Bosscha K, van Berge-Henegouwen GP, van Erpecum KJ (2005): Small gallstones are associated with increased risk of acute pancreatitis: potential benefits of prophylactic cholecystectomy? Am J Gastroenterol 100, 25402550

Villatoro E, Mulla M, Larvin M (2010): Antibiotic therapy for prophylaxis against infection of pancreatic necrosis in acute pancreatitis. Cochrane Database Syst Rev CD002941

Wang G-J, Gao C-F, Wei D, Wang C, Ding S-Q (2009): Acute pancreatitis: etiology and common pathogenesis. World journal of gastroenterology: WJG $\underline{15}, 1427-1430$

Werner J, Uhl W, Buchler MW (2003): Surgical Treatment of Acute Pancreatitis. Curr Treat Options Gastroenterol 6, 359-367

Werner J, Feuerbach S, Uhl W, Buchler MW (2005a): Management of acute pancreatitis: from surgery to interventional intensive care. Gut $\underline{54}, 426-436$

Werner J, Hartwig W, Hackert T, Buchler MW (2005b): Surgery in the treatment of acute pancreatitisopen pancreatic necrosectomy. Scand J Surg $\underline{94}, 130$

Widdison AL, Karanjia ND (1993): Pancreatic infection complicating acute pancreatitis. Br J Surg $\underline{80}$, 148-154

Widdison AL, Karanjia ND, Reber HA (1994): Routes of spread of pathogens into the pancreas in a feline model of acute pancreatitis. Gut $35,1306-1310$

Winter Gasparoto RC, Racy Mde C, De Campos T (2015): Long-term outcomes after acute necrotizing pancreatitis: what happens to the pancreas and to the patient? JOP $\underline{16}, 159-166$

Zhou J, Ke L, Tong Z, Li G, Li W, Li N, Li J (2015): Risk factors and outcome of Splanchnic venous thrombosis in patients with necrotizing acute pancreatitis. Thromb Res $135,68-72$ 


\section{$7 \quad$ Tabellenverzeichnis}

Tabelle 1: Ranson-Score (Ranson 1982) ...................................................................................

Tabelle 2: IKPM der akuten nekrotisierenden Pankreatitis .................................................................15

Tabelle 3: Demographische Daten und Begleiterkrankungen des Gesamtkollektivs sowie der Teilkollektive aus Homburg/Saar und Göttingen 18

Tabelle 4: Ätiologie der Pankreatitis des Gesamtkollektivs sowie der Teilkollektive aus Homburg/Saar und Göttingen

Tabelle 5: Komplikationen und Organersatztherapie des Gesamtkollektivs sowie der Teilkollektive aus Homburg/Saar und Göttingen ...................................................................................20

Tabelle 6: Operative Therapiemaßnahmen des Gesamtkollektivs sowie der Teilkollektive aus Homburg/Saar und Göttingen

Tabelle 7: Perioperative Daten des Gesamtkollektivs sowie der Teilkollektive aus Homburg/Saar und Göttingen

Tabelle 8: Primäre Endpunkte: Krankenhausmortalität sowie 30- und 90-Tages-Mortalitäten des

Gesamtkollektivs sowie der Teilkollektive aus Homburg/Saar und Göttingen

Tabelle 9: Sekundäre Endpunkte des Gesamtkollektivs sowie der Teilkollektive aus Homburg/Saar und

Göttingen

Tabelle 10: Demographische Daten und Komorbiditäten. Subgruppenanalyse: Einfluss der verschiedenen chirurgischen Therapiekonzepte

Tabelle 11: Ätiologie. Subgruppenanalyse: Einfluss der verschiedenen chirurgischen Therapiekonzepte

Tabelle 12: Komplikationen und Organersatztherapie. Subgruppenanalyse: Einfluss der verschiedenen chirurgischen Therapiekonzepte

Tabelle 13: Primäre Endpunkte. Subgruppenanalyse: Einfluss der verschiedenen chirurgischen Therapiekonzepte. .25

Tabelle 14: Sekundäre Endpunkte. Subgruppenanalyse: Einfluss der verschiedenen chirurgischen Therapiekonzepte. .25

Tabelle 15: Primäre Endpunkte. Subgruppenanalyse: Einfluss eines Koloneingriffs .... 26

Tabelle 16: Sekundäre Endpunkte. Subgruppenanalyse: Einfluss eines Koloneingriffs....... 26

Tabelle 17: Primäre Endpunkte. Subgruppenanalyse: Radikales Vorgehen: Einfluss der Kolektomie ...27 
Tabelle 18: Sekundäre Endpunkte. Subgruppenanalyse: Radikales Vorgehen: Einfluss der Kolektomie

Tabelle 19: Primäre Endpunkte. Subgruppenanalyse: Auswirkung der Durchführung einer Kolektomie im Rahmen des Ersteingriffs

Tabelle 20: Sekundäre Endpunkte. Subgruppenanalyse: Auswirkung der Durchführung einer

Kolektomie im Rahmen des Ersteingriffs .28

Tabelle 21: Primäre Endpunkte. Subgruppenanalyse 1: Stellenwert einer frühzeitigen Kolektomie .......29

Tabelle 22: Sekundäre Endpunkte. Subgruppenanalyse 1: Stellenwert einer frühzeitigen Kolektomie ..29

Tabelle 23: Primäre Endpunkte. Subgruppenanalyse 2: Stellenwert einer frühzeitigen Kolektomie .......30

Tabelle 24: Sekundäre Endpunkte. Subgruppenanalyse 2: Stellenwert einer frühzeitigen Kolektomie ..30

Tabelle 25: Primäre Endpunkte. Subgruppenanalyse 1: Zentrumsübergreifende Analyse des

Stellenwertes der Kolektomie

Tabelle 26: Sekundäre Endpunkte. Subgruppenanalyse 1: Zentrumsübergreifende Analyse des

Stellenwertes der Kolektomie

Tabelle 27: Primäre Endpunkte. Subgruppenanalyse 2: Zentrumsübergreifende Analyse des

Stellenwertes der Kolektomie

Tabelle 28: Sekundäre Endpunkte. Subgruppenanalyse 2: Zentrumsübergreifende Analyse des

Stellenwertes der Kolektomie 


\section{Publikationen}

Ein Abstract (Vortrag) zu der Studie mit dem Titel „Management der komplizierten akuten nekrotisierenden Pankreatitis - Stellenwert einer aggressiven chirurgischen Therapie mit Kolektomie“ (Autoren: Jan E. Slotta, Marieke H. Thomsen, B. Michael Ghadimi, Otto Kollmar) wurde bei der Deutschen Gesellschaft für Allgemein- und Viszeralchirurgie (DGAV) für den Viszeralmedizin-Kongress 2015 in Leipzig vom 17.-20.9.2015 eingereicht.

Über die Annahme des Abstracts wurden die Autoren bisher noch nicht informiert. 


\section{Danksagung}

Ich danke Herrn Prof. Dr. Otto Kollmar für die Überlassung des Dissertationsthemas sowie die fachlichen Korrekturen und wertvollen Anregungen während der Durchführung dieser Arbeit. Weiterhin danke ich Herrn Prof. Dr. Otto Kollmar sowie Herrn Dr. Jan E. Slotta für die Hilfe bei der Erweiterung der Datenbank durch die bereits erfassten Patienten aus Homburg/Saar sowie der Bereitstellung der digitalen Intensivakten des Patientenkollektivs des Universitätsklinikums Homburg/Saar.

Herrn Dr. Jan E. Slotta möchte ich für die hervorragende Betreuung, die Unterstützung bei der statistischen Auswertung der Daten und seine ständige Hilfsbereitschaft sehr danken.

Nicht zuletzt gilt ein besonderer Dank Herrn Dr. Uwe Niederberger (CAU Kiel) für Korrekturen, Ideen und fachübergreifende Unterstützung bei der Verfassung dieser Arbeit. 